\title{
O SISTEMA DE FONTES DO DIREITO E A LINGUAGEM NORMATIVA. UMA REFLEXÃO SOBRE A ASSIMETRIA ENTRE PROPOSTAS LEGISLATIVAS E APLICACÃO
}

\author{
THE SYSTEM OF SOURCES OF LAW AND NORMATIVE LANGUAGE. A REFLECTION ON THE \\ ASSYMETRY BETWEEN LEGISLATIVE PROPOSALS AND APPLICATION
}

EL SISTEMA DE FUENTES DEL DERECHO Y EL LENGUAJE NORMATIVO. UNA REFLEXIÓN SOBRE LA ASIMETRIIA ENTRE PROPUESTAS LEGISLATIVAS Y APLICACIÓN

Jacopo Paffarini ${ }^{1}$

1.1 La Costituzione e il linguaggio normativo; 1.2 La disposizione e I'applicazione; 1.3 Il "progetto" e la sua "esecuzione; 1.4 La struttura del discorso: le preposizioni normative e le relazioni tra le fonti; 1.5 L'effettività e la validità del discorso giuridico; 1.6 L'unità dell'ordinamento e l'applicazione delle disposizioni; 1.7 L'interpretazione e il linguaggio normativo; 1.8 L'argomentazione e la decisione; 1.9 L'interpretazione come "scoperta" e come "creazione".

\section{RIASSUNTO}

Una nuova riflessione teorica attorno all'attuale fisionomia dell'esperienza giuridica è imposta dai recenti cambiamenti intervenuti nel sistema delle fonti giuridiche anche a seguito dei processi di integrazione sovranazionale e della crescente interazione tra ordinamenti giuridici di Stati diversi. Il linguaggio giuridico non può essere isolato dal contesto sociale sul quale è destinato a dispiegare i suoi effetti e davanti a delle regole di validità formale e sostanziale che sorreggono la sua formazione, creata dalla necessità pratica che, in ultima istanza, determina il "diritto vivente". La progettualità giuridica, dunque, non si risolve mai nell'ambito della sola fase legislativa ma si estende fino all'attuazione della regola di condotta, momento in cui le categorie della scienza giuridica incontrano quelle dell'etica e delle scienze sociali.

PAROLE CHIAVE: Gerarchia delle fonti. Unità dell'ordinamento giuridico. Interpretazione. Principio di Effettività. Validità della norma.

\section{RESUMO}

Uma nova reflexão teórica no entorno da atual fisionomia da experiência jurídica é imposta pela recente mudança intervinda no sistema de fontes jurídicas que surgiram do processo de integração supranacional

1 Doutor em Direito Público pela Università degli Studi di Perugia (Itália). Mestre em Direito com a colaboração do Centro Tecnológico de Monterrey (Campus Ciudad de México). Bacharel em Direito pela Università degli Studi di Perugia. Pesquisador Visitante do Instituto Max-Planck de Direito Público Comparado - Heidelberg (Alemanha). Membro pesquisador do Grupo Internacional de Pesquisa "Corte, Doutrina e Sociedade Inclusiva: o impacto das fontes doutrinárias nas Cortes Superiores", financiado pelo Ministério de Instrução, das Universidades e Pesquisa (Decreto n. 719/2012). Colaborador assistente na Cátedra de Direito Constitucional e Direito Público Comparado, de titularidade do Prof. Dr. Maurizio Oliviero, junto ao Curso de Direito da Università degli Studi di Perugia. Coorientador do módulo de Direito Transnacional no curso de Direito Público Comparado. Participante do Projeto de Lei Transnacional na London Law School of Economics (Inglaterra). Tem experiência na área de Direito, com ênfase em Direito Público, atuando principalmente nos seguintes temas: direito transnacional, constitucionalismo, direitos humanos, hermenêutica do direito e instituições judiciais. 
e da crescente interação entre ordenamentos jurídicos de Estados diversos. A linguagem jurídica não pode estar isolada do contexto social para a qual é destinada a implantar seus efeitos e ante quais regras de validade formal e substancial que apoia a sua formação, produzida da necessidade prática que, em última instância, determina o "direito vivente". A proposta legislativa, portanto, não se resolve mais no âmbito somente da fase legislativa, mas se estende ao fim da atuação da regra de conduta, momento em que a categoria da ciência jurídica encontra a ética e a ciência social.

PALAVRAS-CHAVE: Hierarquia de fontes. Unidade do ordenamento jurídico. Interpretação. Princípio da efetividade. Validade das normas.

ABSTRACT

A new theoretical reflection on the context of the current physiognomy of legal experience is imposed by the recent change in the system of legal sources that have occurred in the process of supranational integration, and the growing interaction between the legal systems of the various States. Legal language cannot be isolated from the social context in which it will be effective, and in light of which any rules of formal and substantial validity that supports its formation, produced out of a practical need, in the case of the latter, determine the "living law". The legislative proposal, therefore, is no longer resolved in the scope only of legislative phase, but extends to the end of the operation of the rule of conduct, at which moment the category of legal science meets ethics and social science.

KEYWORDS: Hierarchy of sources. Unity of the legal system. Interpretation. Principle of effectiveness. Validity of the norms.

\section{RESUMEN}

Se impone una nueva reflexión teórica en el ámbito de la actual fisonomía de la experiencia jurídica debido a la reciente transformación ocurrida en el sistema de fuentes jurídicas que surgieron del proceso de integración supranacional y de la creciente interacción entre ordenamientos jurídicos de Estados diversos. El lenguaje jurídico no puede estar aislado del contexto social para el cual está destinado a implantar sus efectos y ante cuyas reglas de validez formal y sustancial apoya su formación, producida por la necesidad práctica que, en última instancia, determina el "derecho viviente". La propuesta legislativa, por lo tanto, ya no se resuelve solamente en el ámbito de la fase legislativa, sino que se extiende hasta el fin de la actuación de la regla de conducta, momento en el que la categoría de la ciencia jurídica encuentra a la ética y a la ciencia social.

PALABRAS CLAVE: Jerarquía de fuentes. Unidad del ordenamiento jurídico. Interpretación. Principio de la efectividad. Validez de las normas.

\section{LA COSTITUZIONE E IL LINGUAGGIO NORMATIVO.}

Nel discorso comune, il concetto di Costituzione esprime una fonte di produzione normativa avente una posizione "principale" e "reggente" rispetto all'ordinamento giuridico di un'entità politica (normalmente uno Stato²).

2 Esistono, infatti, Costituzioni che fanno riferimento a comunità nazionali senza Stato (ad esempio quella palestinese). Allo stesso modo, si può dire che, se fosse andato in porto il "Progetto di Costituzione per l'Europa", anche nello spazio giuridico del Vecchio Continente si sarebbe avuta una Costituzione in assenza di uno Stato. 
Un'analisi approfondita della complessa fenomenologia legata al diritto costituzionale, tuttavia, suggerisce di contornare tale concezione con una lunga serie di precisazioni, indispensabili se si vuole dar conto dell'evoluzione che ha interessato la materia.

È empiricamente riscontrabile, nonché abbastanza frequente, il fatto che non ogni singola disposizione della Costituzione riceva attuazione. In altri casi, invece, si è verificato che una previsione costituzionale abbia operato concretamente in modo totalmente diverso rispetto a quanto il testo lasciasse intendere ${ }^{3}$. Infine, è pure possibile che ad una medesima disposizione della Carta siano stati attribuiti significati diversi, e dunque differenti applicazioni, nel corso degli anni.

In questa "distanza" tra la norma scritta e la sua attuazione, del resto, risiede una delle più notevoli caratteristiche del diritto in generale ${ }^{4}$. Lo studio del diritto costituzionale in questo non fa eccezione: come ogni altra fonte di produzione, la Costituzione è, anzitutto, un atto linguistico ${ }^{5}$.

Il linguaggio normativo costituisce pertanto il primo oggetto di studio della scienza giuridica ed acquista una sua peculiarità rispetto al linguaggio descrittivo delle scienze naturali sotto due profili: quello pragmatico e quello semantico ${ }^{6}$. Non costituisce, invece, un valido criterio di identificazione degli enunciati giuridici la forma sintattica (indicativa, imperativa o prescrittiva) della preposizione, dal momento che «gli enunciati indicativi hanno tipicamente, ma non necessariamente, forma indicativa; e gli enunciati prescrittivi hanno tipicamente, ma non necessariamente, forma deontica»`.

L'unico modo certo per poter qualificare un atto linguistico come espressione del "linguaggio prescrittivo", invero, è quello di guardare al suo concreto utilizzo. In questo senso, «descrittivo o prescrittivo è non l'enunciato in quanto tale (type-sentence), ma il suo uso, la sua concreta enunciazione (token-sentence) il suo proferimento (utterance) da parte di qualcuno in circostanze date».

Tuttavia, gli enunciati prescrittivi si contraddistinguono anche dal punto di vista semantico, in quanto esprimono un "dover essere". Il loro significato si risolve in una norma non nella descrizione di un fatto: è"normativo" I'enunciato che può essere "eseguito o non eseguito", non I'enunciato di cui si possa dire se sia "vero o falso".

Da queste premesse possiamo già avanzare una prima osservazione, ossia che il "diritto" si presenti «non proprio come l'insieme delle leggi, ma piuttosto come il contenuto normativo o prescrittivo delle leggi: ciò che le leggi dicono, il loro significato»?

In questo senso, si può e si deve dire che la Costituzione sia un "fatto normativo", ma cionondimeno si ritiene qui lo studio delle dinamiche del diritto costituzionale, e in generale di tutto il diritto, prendano necessariamente piede dall'analisi del linguaggio normativo.

L'esistenza di una prescrizione presuppone che vi sia un destinatario: il soggetto che detiene I'autorità normativa progetta una regola nella sua mente e, attraverso le parole, la comunica ad una o più persone. La comunicazione è utile se và a "buon fine", ossia se colui che intende trasmettere

3 Il potere di emanare decreti legge, ad esempio, è conferito al Governo dall'art.77 solo «in casi straordinari di necessità ed urgenza», tuttavia questa condizione non viene rispettata nella stragrande maggioranza delle ipotesi in cui tale strumento viene utilizzato dall'Esecutivo.

4 A proposito della distanza tra il momento dell'individuazione della norma da applicare al caso concreto e quello della sua materiale realizzazione, è stato osservato che «l'ipotesi della perfetta corrispondenza fra il comportamento previsto ed il comportamento realizzato rappresenta un caso limite, una sorta di infinito ove i valori positivi e negativi si confondono», vedi. A. PIZZORUSSO. Corso di diritto comparato, Milano, 1983, p. 10.

5 Ça va sans dire, i "fatti normativi" (come ad esempio la consuetudine e le fonti extra ordinem), dal momento che non trovano il loro fondamento in atti di volontà, quanto piuttosto nell'effettiva osservanza ed accettazione di una regola da parte dei destinatari, producono diritto senza necessità del medium linguistico.

6 Cfr. R. GUASTINI. Le fonti del diritto. Fondamenti teorici, Milano, 2010, p. 4 e ss.

7 Cfr. R. GUASTINI. Il diritto come linguaggio. Lezioni, Torino, 2006, p. 8. Di fatto, una notevole quantità di disposizioni normative presenta una forma sintattica indicativa: un esempio, tra i tanti possibili, è rinvenibile nell'art. 575 del codice penale, il quale senza dubbio esprime una prescrizione e, come tale, può essere riformulato in una forma sintattica deontica. Sul punto si veda anche R. GUASTINI. Le fonti del diritto, cit., p. 5: «non vi è alcuna corrispondenza biunivoca tra la forma sintattica degli enunciati e la loro funzione, rispettivamente, descrittiva o prescrittiva».

$8 \quad I v i$, p. 7.

$9 \quad$ IVi, p. 3. 
il messaggio viene effettivamente compreso da coloro con i quali interagisce. Ciò talvolta avviene, mentre in altri casi uno o più di uno o tutti i destinatari non capiscono cosa si intende comunicare, oppure ancora, capiscono qualcosa di diverso da ciò che colui che comunica vuol far intendere ${ }^{10}$. A tal proposito, si parla di "discorso giuridico", in quanto i soggetti che interagiscono (siano essi in una posizione di parità o meno) utilizzano e/o discutono su enunciati dell'autorità normativa.

L'interazione e la comprensione tra comunicanti è resa possibile dal fatto che il "discorso" presuppone «un corpo coerente di preposizioni astratte che implica una logica, un ordine e la possibilità non solo di esistere ma soprattutto di riprodursi, di svilupparsi secondo leggi interne alla stessa logica» ${ }^{11}$.

Osservando la dimensione semantica dell'atto normativo, dunque, non possiamo fare a meno di considerare separatamente "parole" (ossia ciò che ci descrive la presenza di una norma) e "contenuto" (ossia la norma vera e propria). Sotto questa prospettiva, si possono dedurre due prime caratteristiche costanti del discorso giuridico, I'una relativa al piano "strutturale", ossia la distinzione tra disposizioni e norme ${ }^{12}$, I'altra di livello "pragmatico-applicativo", ossia il processo di «specificazione» del significato dell'enunciato giuridico, il quale viene comunemente chiamato "interpretazione"13.

Prima di approfondire questi aspetti, è opportuno, tuttavia, fare una serie di considerazioni preliminari circa la natura, o meglio l'origine, delle "preposizioni giuridiche".

In primo luogo, in quanto "atti" linguistici, gli enunciati normativi tendono ad uno scopo ${ }^{14}$, quello di prescrivere, ossia ordinare alcunché costituente un comportamento attivo od omissivo. Il diritto, pertanto, è sempre rivolto ad esseri umani: anche qualora I'oggetto degli enunciati non sia un'azione od un'omissione, ma, piuttosto, un risultato (ad esempio, proteggere i minori, dichiarare la nullità di un contratto, ecc...) è sempre mediante una qualche condotta umana (di una persona fisica, giuridica o del titolare di un organo) che l'esito comandato può essere realizzato. L'origine del diritto spiega dunque il suo scopo pratico. Invero, è stato detto che «il diritto si risolva in un complesso di norme mediante le quali i comportamenti umani posti in essere nell'ambito di una determinata società vengono in vario modo incoraggiati o scoraggiati» ${ }^{15}$, con l'intento di impedire che essi siano totalmente rimessi alla fatalità delle circostanze. La convivenza genera la necessità di una regolazione, il diritto ha la funzione di determinare il "progetto" all'interno del quale si svolgono le condotte dei componenti di una determinata organizzazione sociale. Un progetto del quale, tra l'altro, viene teorizzata anche la "completezza", in quanto si presume che il discorso giuridico utilizzi una lingua "chiusa", alla stregua del discorso matematico e delle scienze formali: la "norma di chiusura" del sistema sarebbe data infatti dalla prescrizione (inespressa, ma secondo alcuni ricavabile in via logica ${ }^{16}$ ) per la quale "tutto ciò che non è vietato è permesso" all'individuo ${ }^{17}$.

In secondo luogo, essendo il diritto orientato ad un qualche scopo individuato dai consociati o, più spesso, da coloro che sono titolari dei poteri normativi, necessariamente presuppone un

10 Cfr. G.U. RESCIGNO. Comunicare, comprendere interpretare nel diritto, in Diritto pubblico, n.3/2009, p. 694.

11 Vedi M. MIAILLE. Introduzione allo studio critico del diritto, Roma, 1979, p. 23.

12 Con il termine "disposizione" intenderemo «ogni enunciato appartenente ad una fonte del diritto» Per "norma", invece, si intenderà «il suo contenuto di senso, il significato, che è una variabile dipendente dell'interpretazione». Vedi. R. GUASTINI, Le fonti del diritto, cit., p. 36.

13 Cfr. A. PIZZORUSSO, Corso di Diritto Comparato, cit., p. 10. Si veda anche N. BOBBIO, Scienza del diritto e analisi del linguaggio(1950), oggi in Contributo ad un dizionario giuridico, Torino, 1994, p. 348 , per il quale se, da un lato, è vero che «la materia su cui opera il giurista è un complesso di regole di comportamento», dall'altro, «questo può compiere due ricerche distinte, $[\ldots .]:$.0 analizzare la regola in quanto tale, oppure il contenuto della regola, cioè il regolato». Attraverso il primo tipo di studio, il giurista contribuisce alla formazione di quella che viene definita "teoria generale del diritto" (che secondo I'A., andrebbe più propriamente definita "teoria formale"); lo studio del "contenuto" della regola costituisce invece l'oggetto di ricerca della "giurisprudenza".

14 «Sono linguistici tutti quegli atti che si compiono mediante il linguaggio, che si eseguono" a parole"», vedi. R. GUASTINI. Le fonti del diritto, cit., p. 6.

15 Vedi A. PIZZORUSSO. Corso di diritto comparato, cit., p. 8.

16 Cfr. J. RAZ. Legal reasons, sources and gaps, in The authority of law, Oxford, 1979, p. 75 e ss.

17 Sulla differenza tra "completezza" e "completabilità"del linguaggio normativo si rinvia a N. BOBBIO. II positivismo giuridico, Torino, 1961. Cfr., inoltre, dello stesso Autore, Scienza del diritto e analisi del linguaggio, cit., p. 356 e ss. 
momento decisionale, nell'ambito del quale il linguaggio normativo prende forma. Anche sotto questo punto di vista, l'esistenza ed il contenuto del diritto in una società dipende da fatti sociali, ossia dai comportamenti e dalle attività dei soggetti legittimati alla produzione giuridica. L'autorità del diritto, pertanto, «dipende dal fatto che si tratta di un mezzo convenzionalmente accettato per produrre conseguenze sociali desiderabili» ${ }^{18}$ in risposta ad una domanda di giustizia proveniente da una determinata comunità.

Per il momento, si ritiene opportuno limitarci a queste premesse, astenendoci dal fare ulteriori considerazioni in merito ai contenuti normativi dell'ordinamento, ossia circa il diritto "come è" e come, invece, "dovrebbe essere".

\section{LA DISPOSIZIONE E L'APPLICAZIONE.}

Il concreto funzionamento delle norme giuridiche, come si è detto, può essere meglio compreso se si tiene conto della distanza che esiste tra il momento della creazione delle norme da parte del legislatore e quello della loro applicazione alle fattispecie concretamente realizzatesi

$\mathrm{E}^{\prime}$ stato osservato che il tempo è parte costitutiva dell'«esperienza giuridica» ${ }^{19} \mathrm{e}$ che il discorso giuridico tragga da questa pur semplice constatazione una delle sue più importanti caratteristiche: la progettualità.

Come abbiamo sopra osservato, un atto linguistico prescrittivo esprime un comando il quale può essere soltanto eseguito o meno. Abbiamo, inoltre, stabilito che la sintassi non è un elemento in grado di identificare le enunciazioni prescrittive rispetto a quelle descrittive, dato che talvolta anche preposizioni non aventi forma imperativa possono assumere veste normativa. Abbiamo identificato, pertanto, come "prescrizione" o "norma", il contenuto semantico, ossia il significato, di tutte quelle enunciazioni che, di fatto, vengono usate per compiere l'atto di prescrivere.

Ora, ogni fonte del diritto, come preposizione linguistica, si può costruire in diversi modi, ossia contenere i più diversi predicati, riferimenti fattuali e temporali, ecc... Tuttavia, le regole di comportamento, ossia il contenuto prescrittivo che le preposizioni normative esprimono, possono essere inquadrate secondo degli schemi di costruzione ben definiti, in cui distinguiamo delle "parti" necessarie ed altre la cui presenza è solo eventuale ${ }^{20}$. Si può così notare uno "schema base" composto da tre parti la cui presenza è essenziale per avere un enunciato giuridico di senso compiuto: il soggetto o la categoria di soggetti $(X)$ dalla quale si pretende il comportamento; I'indicazione della «modalità deontica» (obbligatorio, vietato o permesso); la descrizione del comportamento oggetto di prescrizione $(\mathrm{f})^{21}$. Qualora le circostanze lo richiedano, tuttavia, I'autorità normativa potrà anche indicare i destinatari del comportamento (B), le circostanze di tempo (t) e di luogo (I) della condotta prescritta. La regola di comportamento, a questo punto avrà raggiunto il suo "schema completo" che si può esprimere nel modo seguente: "X deve, deve non, può fare $f$ nei confronti di B nel periodo t e nel luogo I".

Ricollegandoci al discorso che è stato fatto in apertura, possiamo dedurre che la veste formale del linguaggio normativo potrà variare, di volta in volta, a seconda della quantità di comportamenti che ci si propone di comandare. Così, ad esempio, lo schema completo della regola di comportamento, come è stato sopra descritto, invierà un comando puntale la cui "operatività" si esaurisce in circostanze di fatto (persone, luoghi, comportamenti,...) e di tempo ben definite. Lo "schema base", invece, delinea un progetto di comportamento la cui esecuzione potrà avvenire un numero indefinito di volte, verso un numero indefinito di persone e in un numero indefinito di luoghi.

A ciò si aggiunga che, insieme alla struttura dell'enunciazione, possono variare i suoi destinatari (i soggetti attivi della condotta) nonché la descrizione del comportamento ${ }^{22}$. Riguardo al primo profilo avremo una regola particolare o generale a seconda che sia indirizzata ad un numero già determinato

18 Vedi J. L. COLEMAN. On the relationship between laws and morals, in Ratio juris, 2, 1989, pp. 66 e ss.

19 Cfr. G.U. RESCIGNO. L'atto normativo. Bologna, 1998, p. 2.

20 Ivi, p. 12.

21 In tal modo, si può individuare nell'espressione "X deve/deve non/ può fare f" I'esempio più elementare di regola giuridica che può essere fatto.

22 Ivi, p. 14. 
o, comunque, determinabile ${ }^{23}$ di persone, oppure che l'insieme dei destinatari sia "aperto" e non definibile a priori (ad esempio, "i cittadini", "i lavoratori", "le madri", ecc...). Sotto il secondo profilo, invece, una norma potrà essere definita concreta o astratta a seconda che il comportamento che si vuole prescrivere sia determinato e precisamente collocato nel tempo e nello spazio oppure sia indicata solamente la categoria di azioni od omissioni che si vuole comandare. Nella prassi giuridica, inoltre, queste quattro polarità tendono a combinarsi tra loro dando vita ad altrettante classi di regole di comportamento.

Ora, sebbene gli atti linguistici appartenenti alle classi di regole così delimitate costituiscano senza dubbio degli enunciati prescrittivi, non tutti potrebbero essere definiti "atti normativi". Nel discorso giuridico, così come in quello comune, quando si parla di "norme", si è soliti riferirsi ad enunciazioni relative a fattispecie astratte e generali24: a partire dalla nascita del costituzionalismo l'atto normativo per eccellenza è la "legge", attraverso la quale si esprime la volontà dei rappresentanti del corpo elettorale che siedono in Parlamento. Se si assume questa prospettiva, si comprende il motivo per cui si è insistito sull'elemento temporale e programmatico che l'atto normativo esprime rispetto al "presente" del corpo sociale. Utilizzando il termine "progetto" dunque presupponiamo, da un lato, che una "esecuzione" prima o poi avvenga, dall'altro, di trovarci in una fase temporalmente antecedente ad essa, ossia al verificarsi del caso concreto che diventerà oggetto della disciplina normativa.

Ora, è logicamente deducibile, nonché riscontrabile nella pratica giuridica, che quando l'autorità normativa emette regole di comportamento particolari e concretizza la fattispecie che si intende disciplinare si sia già verificata o si stia verificando nella pratica. Ad esempio, quando la Pubblica Amministrazione si esprime con enunciazioni del tipo "Il soggetto $x$, poiché commerciante privo di licenza, deve pagare l'ammenda y entro il giorno $t$ ", si vuole indirizzare il comportamento umano verso un risultato ben preciso: non siamo di fronte ad un "progetto razionale" che anticipa una serie indefinita di comportamenti, ma ad una semplice richiesta di esecuzione.

Gli attributi di astrattezza e di generalità, dunque, ci permettono di distinguere le "norme" dai "provvedimenti": le prime avrebbero un contenuto "dispositivo" e costituiscono la base motivazionale dei secondi, che hanno invece un contenuto limitatamente "imperativo". Quando i soggetti della regola sono definiti, così come la forma, il luogo e il tempo del comportamento prescritto, I'atto dell'autorità giuridica è un "provvedere", ossia «decidere ex post facto in relazione a precise circostanze già verificatesi». All'opposto, «disporre significa stabilire la conseguenza giuridica di una fattispecie generale ed astratta, ossia decidere ex ante in relazione ad una classe di fattispecie future, di cui per ciò stesso si ipotizza (e non: si costata) I'occorrenza» ${ }^{25}$. Il rapporto di strumentalità che lega le norme ai provvedimenti è deducibile dal fatto che, la motivazione che normalmente compare nella formulazione scritta di questi ultimi, riporta, esplicitamente o implicitamente, la fonte normativa dalla quale può essere astratta la regola di condotta applicata al caso concreto.

Ricollegandoci a quanto visto sopra, si può affermare che l'atto normativo contiene non già una "norma" (o "regola di comportamento"), ma una "disposizione normativa", intendendosi piuttosto con la prima il contenuto di significato da essa espresso.

Il carattere generale ed astratto delle norme giuridiche, secondo un'ulteriore ricostruzione, permette che esse possano essere sempre tradotte in enunciati a struttura condizionale o ipotetica ${ }^{26}$. In tal senso, nella formulazione della norma giuridica potrà sempre distinguersi un "antecedente" 23 Nel senso che i soggetti attivi della condotta siano individuabili uno ad uno.

$24 \mathrm{Si}$ esclude, pertanto, che le regole particolari e concrete possano essere espresse da atti normativi. Rispetto alle due classi intermedie che possono sorgere dalla combinazione delle due precedenti polarità (regole generali e concrete, regole particolari ed astratte) non si riscontra uniformità di opinioni in dottrina. Tuttavia, seguendo l'orientamento della letteratura da ultimo citata, sembra che l'elemento di distinzione determinante possa essere rintracciato nella tipologia di comportamento prescritto. Pertanto, gli atti che esprimono regole generali e concrete, sebbene si riferiscano ad una quantità di soggetti indeterminata, non possono essere definiti "normativi" dato che i comportamenti sono tutti previamente determinati; viceversa, gli atti particolari ed astratti «vengono sentiti come normativi». Ivi, p. 15.

25 Vedi R. GUASTINI. Le fonti del diritto, cit., p. 17. Cfr. anche V. CRISAFULLI, Atto normativo, in Enciclopedia del diritto, vol. IV, Milano, 1959, p. 255.

26 Tra i molti: Cfr. R. GUASTINI. Le fonti del diritto, cit., p. 15; G.U. RESCIGNO. L'atto normativo, cit., p. 14. Tra gli autori stranieri, cfr. E. BULYGIN, C.E. ALCHOURRON. La norma giuridica, in E. BULYGIN, Il positivismo giuridico,Milano, 2007, p. 217. 
ed un "conseguente": il primo individua la condizione (ossia gli attributi della fattispecie astratta), il secondo la conseguenza giuridica (la liceità o la doverosità di un comportamento ${ }^{27}$ ). Ovviamente, entrambi gli elementi costitutivi dovranno possedere il carattere dell'astrattezza: non una circostanza ed una conseguenza, ma una classe di circostanze ed una classe di conseguenze. In caso contrario non si avrebbe una regola ipotetica o condizionale, bensi "categorica" in quanto relativa ad un "caso individuale"28. In questo senso, «la grande maggioranza delle norme giuridiche sono condizionali; certamente sono condizionali tutte le norme generali. Solo tra le norme giuridiche individuali (come, ad esempio, le sentenze giudiziali) si possono incontrare norme categoriche» ${ }^{29}$.

Ritornando alla distinzione tra "disporre" e "provvedere", così come è stata sopra delineata, si ribadisce che generalmente le norme ipotetiche espresse dagli atti normativi fungono da presupposto per l'emanazione di norme categoriche. Così nel discorso giuridico, chi pone la norma ipotetica "se $A$ allora $B$ ", stabilisce le premesse, nonché la motivazione, del proseguimento del discorso attraverso il provvedimento "poiché $A$ allora $B$ ". La diversa struttura logica degli enunciati, conferma la differente attività che nei due casi sarebbe posta in essere dall'autorità normativa: laddove nel primo caso progetta una serie indefinita di comportamenti futuri, nel secondo constata il verificarsi delle circostanze e provvede in concreto secondo quanto ha prescritto la norma.

\section{IL "PROGETTO" E LA SUA "ESECUZIONE".}

Tra le regole generali ed astratte espresse dagli atti normativi e le regole concrete che trovano applicazione nei casi individuali non vi è un rapporto unico: come si è visto, l'esperienza giuridica nel suo complesso è scandita in ogni suo momento da differenti "livelli operativi". Alcuni si collocano nell'ambito della "scala della progettazione" normativa e, dunque, interessano i rapporti tra atti normativi; gli altri riguardano I"attuazione" di ciascuna scala del progetto, ossia I'applicazione delle disposizioni contenute negli atti normativi. Sotto il primo profilo si possono ricondurre i passaggi che operano «sul versante della formazione delle norme», ossia «quelli che consentono di passare da norme più generali a norme progressivamente più dettagliate, come ad esempio avviene quando una norma costituzionale è specificata ed attuata mediante una legge, oppure quando una legge è specificata ed attuata mediante un regolamento di esecuzione» ${ }^{30}$. Il secondo aspetto, ricomprenderà invece le più diverse ipotesi nelle quali l'operatore giuridico o i privati si trovino ad interpretare una qualsiasi norma giuridica.

La norma giuridica esprime un progetto di comportamento, ma a sua volta trova fondamento in un'altra della quale dovrà "rispettare" ed "eseguire" il contenuto. Così se ci si muove in senso "ascendente", a partire dalla regola-provvedimento particolare e concreta, si delinea una scala crescente di progetti la cui generalità ed astrattezza mano a mano si fa sempre più accentuata.

In tal senso, la definizione di Costituzione come "Norma Fondamentale" non dipende soltanto dal fatto che essa esprime i principi ed i valori in base ai quali una comunità ha scelto di convivere, quanto piuttosto dal fatto che essa necessariamente «esprime il progetto di massima, quello che viene per primo e disegna le grandi linee; vi saranno poi altri progetti, a scala sempre minore, fino a quel progetto operativo che ha bisogno solo di essere tradotto in concreti comportamenti» ${ }^{31}$.

Di seguito si parlerà delle caratteristiche del testo costituzionale e delle principali peculiarità che caratterizzano "I'origine" della costituzione rispetto a quella degli altri atti normativi, tuttavia, quello che ora interessa puntualizzare è che in ogni passaggio da una scala del progetto all'altra

27 Questa ricostruzione può essere utilizzata, come si vedrà, anche per le norme giuridiche il cui "conseguente" non si identifica con la qualificazione deontica di un comportamento, ma stabilisce un determinato effetto giuridico in relazione ad una fattispecie determinata. Ad esempio, le norme che qualificano valido o invalido un contratto in determinate circostanze, quelle che definiscono le condizioni per l'acquisto di un determinato status civile, ecc...

28 Altresì, una regola di comportamento "particolare e concreta" nel senso che si è prima spiegato. Dunque, «se l'obbligo (o il divieto o il permesso) stabilito non è soggetto ad alcuna condizione, allora la norma è categorica», vedi. E. BULYGIN, C.E. ALCHOURRON. La norma giuridica, cit., p. 227.

29 Ivi, p. 227.

30 Vedi A. PIZZORUSSO. Corso di diritto comparato, cit., p. 10.

31 Vedi G.U. RESCIGNO. La Costituzione come progetto apicale e generale, in Le fonti del diritto, oggi. Giornate di studio in onore di Alessandro Pizzorusso, Pisa, 2006, p. 32. 
coesistono due elementi: un "enunciato" ed una "decisione". Il primo esprime quello che viene comunemente definito in dottrina "diritto teorico" e costituisce l'oggetto dell'attività di interpretazione. Dall'altra parte, invece, la decisione dà corpo alla nozione di "diritto vivente", ossia «il complesso delle norme che in un dato momento storico hanno trovato attuazione mediante i comportamenti degli operatori» ${ }^{32}$, in altre parole, il risultato (storico) dell'attività interpretativa ed applicativa. La distinzione, oltre ad avere una finalità ricostruttiva della materia giuridica, ha un fondamentale rilievo pratico alla luce del fatto che normalmente o forse sempre, ma non è un problema che ora si vuole affrontare «ad ogni disposizione corrispondono più norme congiuntamente» ${ }^{33}$.

Ricapitolando, il linguaggio normativo assume rilevanza sotto un duplice profilo.

Dal punto di vista strutturale, infatti, il "discorso giuridico" si articola in una serie di enunciati normativi, ciascuno dei quali riceve attuazione nel comportamento degli operatori giuridici, dalle autorità pubbliche fino ai privati. Organizzandosi in vari "livelli" (esattamente come il sistema giuridico), il discorso giuridico può essere più o meno "lungo" a seconda del "punto di partenza", ossia del grado di astrattezza, rispetto al caso concreto, della norma selezionata dall'operatore giuridico o, comunque, del numero di "passaggi" che vengono eseguiti nel processo di "specificazione" ${ }^{134}$. Può darsi anche il caso che la regola generale ed astratta sia di per sé rispettata senza necessità di passare ad una individuale, come ad esempio, si può riscontrare con la generale osservanza delle norme del codice penale: in caso di violazione, tuttavia, la sentenza di condanna rappresenterà il "progetto conclusivo" del discorso giuridico, al quale può seguire solo l'esecuzione. L'estrema semplificazione qui compiuta nasconde la natura reale del rapporto tra la regola generale ed astratta e la regola individuale che, invero, non è mai un rapporto univoco, ma sempre un rapporto "uno-molti": davanti ad ogni caso concreto, la regola particolare verrà "costruita" di volta in volta mediante il collegamento tra più enunciati ognuno dei quali svolgerà un ruolo proprio nel discorso ${ }^{35}$.

La "formazione" della regola di comportamento sulla quale si basa la "decisione" del caso generalmente viene "argomentata" in vario modo da parte dell'operatore giuridico. In ambito processuale, tuttavia, data l'immediata importanza "pratica" che assume il provvedimento finale del giudice, la questione della motivazione assume dei contorni più definiti e costanti. È possibile, infatti, distinguere due aspetti differenti del ragionamento del giudice: il primo attiene alla c.d. giustificazione interna della decisione, ossia all'esposizione dei profili "di fatto" (premesse conoscitive) e "di diritto" (premesse normative) relativi al caso e che il giudice provvederà a "ordinare" in maniera tale che «il contenuto della decisione [possa] essere logicamente dedotto da una norma in congiunzione con una preposizione conoscitiva» ${ }^{36}$; il secondo, la c.d. giustificazione esterna, attiene alla scelta delle "premesse normative" ("Se F allora C") e "conoscitive" (I'insieme di preposizioni in base alle

32 Vedi A PIZZORUSSO. Corso di diritto comparato, cit., p. 13.

33 Così R. GUASTINI. Le fonti del diritto, cit., p. 36.

$34 \mathrm{Si} \mathrm{veda,} \mathrm{in} \mathrm{tal} \mathrm{senso,} \mathrm{G.U.} \mathrm{RESCIGNO.} \mathrm{L'atto} \mathrm{normativo,} \mathrm{cit.,} \mathrm{p.} \mathrm{43,} \mathrm{per} \mathrm{il} \mathrm{quale} \mathrm{«tra} \mathrm{le} \mathrm{tante} \mathrm{ragioni}$ per cui il sistema giuridico merita il nome di sistema e cioè di insieme strutturato, vi è anche quella che richiama il fatto che il sistema giuridico si articola in livelli, ed egualmente in livelli si articola qualsiasi discorso giuridico. Quali e quanti poi sono tali livelli, dipende dal discorso che viene costruito o considerato: ogni volta daccapo gli esseri umani strutturano secondo i livelli appropriati al caso il loro discorso giuridico. Ciò che in un discorso appare di primo livello può diventare di secondo in un altro discorso, e viceversa».

35 Ivi, p. 43.

36 Cfr. R. GUASTINI. Il diritto come linguaggio, cit., p. 198. È opportuno precisare, come sostiene I'A., che I'insieme delle preposizioni della motivazione della sentenza che costituiscono la c.d. giustificazione interna hanno lo scopo di conferire «validità logica» alla conclusione, la quale è cosa ben diversa dalla «fondatezza giuridica». Quando il giudice enuncia il dispositivo, infatti, si ritiene che sia già stata definita la "qualificazione giuridica" dei fatti oggetto della controversia e, pertanto, l'eventuale sussunzione della fattispecie concreta nella categoria astratta descritta dalla disposizione normativa. Il giudice, a meno che non intenda "introdurre" dissociazioni non previste dalla lettera della norma e non deducibili dal contesto normativo (nel modo che si vedrà in seguito), non può far altro che applicare le conseguenze giuridiche stabilite dalla regola presa in considerazione, riproducendo un ragionamento di tipo logico-deduttivo. La «fondatezza giuridica» della decisione riguarda, invece, la validità delle "premesse normative" (ossia, delle regole di comportamento a cui si dà applicazione con il dispositivo della sentenza) e la verità delle "premesse conoscitive" (ossia degli enunciati descrittivi dei fatti oggetto di giudizio). La «fondatezza giuridica» del dispositivo costituisce, come vedremo, I'oggetto delle argomentazioni riconducibili alla "giustificazione esterna" della decisione. 
quali il giudice decide di ricondurre un determinato evento sotto la descrizione normativa " $F$ "), di cui devono essere argomentate, rispettivamente, la "validità" e la "verità".

Sotto un diverso punto di vista - quello interpretativo - I'operatore giuridico è tenuto ad analizzare il linguaggio normativo affinché esso possa essere "messo in atto": questo compito accomuna il lavoro non solo degli "organi dell'applicazione", ma anche di quelli "della produzione normativa" e, ovviamente, dei privati. Ogni enunciato infatti viene, a seconda dei casi, o specificato attraverso un atto normativo (si pensi alle leggi di interpretazione autentica o ad una legge che dà attuazione ad un'altra anteriore ma di portata più generale), o applicato direttamente alla fattispecie concreta. In ambedue le ipotesi, il risultato dell'operazione (sia essa una regola generale ed astratta sia essa una regola di comportamento concreta e puntuale) presuppone un'attività interpretativa. La differenza sta nel fatto che, mentre nel secondo caso segue l'esecuzione, nel primo il discorso giuridico è destinato a proseguire e quindi sarà richiesta un'ulteriore interpretazione.

Nell'esperienza giuridica, dunque, la distanza tra una previsione normativa astratta e il comportamento concretamente attuato è colmata da una serie di "fasi intermedie" in cui «ci possiamo trovare di fronte ad altrettante coppie di disposizioni e norme, la norma elaborata nella precedente fase costituendo la disposizione da applicare nella fase successiva» ${ }^{37}$. Ciò in quanto l'atto normativo, come comportamento linguistico, non può fare a meno di contenere "disposizioni", e ciascuna di queste deve essere interpretata per poter produrre effetti in ordine alla definizione della regola di comportamento relativa al caso concreto. Da ciò si possono dedurre due conseguenze: in primo luogo, richiamando Kelsen, si deve affermare che ogni atto normativo sia al contempo un atto di applicazione (in quanto comportamento linguistico che segue una precedente norma giuridica) e un atto di creazione del diritto (in quanto si risolve nella formulazione di una nuova proposizione giuridica, generalmente di contenuto più specifico). In secondo luogo, l'eventualità che possano esistere una serie di "fasi intermedie" tra previsione ed attuazione, fa si che la norma giuridica (generale ed astratta) a cui si dà esecuzione, possa essere identificata, alternativamente, con il significato, ossia il contenuto di senso, di un singolo enunciato normativo o di un segmento di enunciati normativi o di una combinazione di enunciati normativi ${ }^{38}$.

\section{LA STRUTTURA DEL DISCORSO: LE PREPOSIZIONI NORMATIVE E LE RELAZIONI TRA LE FONTI.}

Un linguaggio si ritiene "rigoroso" quando siano state fissate ed accettate dai comunicanti le regole che presiedono la "formazione" del discorso (ossia le modalità in cui possono essere utilizzate le parole e gli enunciati che di quel linguaggio fanno parte), nonché le regole che presiedono la sua "riproduzione" (ossia le condizioni in cui è "valido" ricavare preposizioni "derivate" a partire da quelle "originarie") $)^{39}$.

Come è già stato anticipato, ogni discorso possiede una propria "logica" e coloro i quali vogliono interagire non possono far altro che "condividerne" le regole costitutive, se vogliono comunicare comprendendosi. La rigorosità del linguaggio, come è stato autorevolmente illustrato ${ }^{40}$, non determina la verità "oggettiva" di ciò che viene affermato, quanto piuttosto la sua valenza "intersoggettiva", cioè la possibilità che il contenuto della comunicazione venga compreso col minor numero di fraintendimenti grazie all'impiego di "concetti" le cui "regole di utilizzo" sono state previamente stabilite ${ }^{41}$.

Il linguaggio normativo, come si è visto, presuppone l'esistenza di un'organizzazione sociale, ossia di una pluralità di soggetti di cui si vuole disciplinare la "vita in comune". Invero, nel loro complesso, gli enunciati che formano l'ordinamento sono "organizzati" con lo scopo di garantire la produzione continua di regole di comportamento. Anzitutto si considerino le disposizioni che si rivolgono a coloro che, in quanto detentori del potere politico, presiedono I'attività di creazione delle norme generali. Il

37 Vedi A PIZZORUSSO. Corso di diritto comparato, cit., p. 16.

38 Cfr. R. GUASTINI. Le fonti del diritto, cit., p. 35.

39 Cfr. N. BOBBIO. Scienza del diritto e analisi del linguaggio, cit., p. 345-346.

40 Ibidem.

41 Ivi, p. 352: «[...] il concetto non è una copia della realtà, ma un mezzo di comunicazione [...]. Quindi un concetto non è più o meno vero, ma più o meno utilizzabile a seconda del maggiore o minore rigore usato nello stabilire le sue regole d'uso». 
diritto progetta, infatti, anche il "comportamento" delle istituzioni pubbliche: definisce i poteri degli organi, le procedure di elezione o di nomina, il funzionamento, ecc... Questo complesso di regole normalmente viene identificato con il "diritto pubblico" delle organizzazioni statali ed assumerà forme e contenuti diversi a seconda degli ordinamenti che si prendono in considerazione.

Ripartendo dai concetti sopra definiti, questo rilievo ci permette di dedurre che non tutti gli enunciati che formano il discorso giuridico esprimono un obbligo o un divieto o un permesso, ma assumono anche altri contenuti di vario genere. La qualificazione deontica di un comportamento, dunque, si può rappresentare come l'atto finale, I'ultima fase di una progettazione che trova il suo apice e punto di partenza nella Costituzione.

Abbiamo detto che la forma sintattica delle proposizioni normative non è condizione sufficiente per I'individuazione di un enunciato prescrittivo e che, pertanto, l'unico criterio certo è dato dell'effettiva esistenza di un "momento applicativo". Tuttavia, dal momento che ogni sistema giuridico disciplina autonomamente la propria "riproduzione", non si potrà non tener conto anche di quelle disposizioni che non esprimono regole di comportamento, ma forniscono le "premesse" e le "condizioni" per il loro funzionamento. Questo tipo di enunciati ricoprono un ruolo assai importante nel discorso giuridico, in quanto, combinandosi tra loro, danno giustificazione alla scelta di ricondurre una fattispecie concreta ad una determinata regola di comportamento. Le modalità in cui le combinazioni possono avvenire sono illimitate e trovano spiegazione, come si è visto, nelle argomentazioni utilizzate dall'operatore giuridico (giudice, Pubblica Amministrazione, privati, ecc...) rispetto alle circostanze che caratterizzano il caso.

Più avanti si tornerà sulla questione della motivazione delle scelte interpretative. Ora si tenga conto che, per comodità espositiva, la parola "norma" verrà utilizzata nel suo significato più ampio, ossia come enunciato appartenente al discorso normativo a prescindere dal fatto che essa abbia un contenuto prescrittivo di un certo comportamento o meno. Esula dallo scopo della presente trattazione offrire una classificazione esauriente delle varie tipologie di enunciati (anche perché molteplici sono le ricostruzioni offerte dalla dottrina a riguardo), bensì ci si limita a considerare quelli che nel complesso risultano per noi più importanti.

In primo luogo, gli enunciati che esprimono le "regole costitutive", le quali ricollegano ad un determinato fatto, anziché un giudizio deontico, la capacità di produrre un determinato effetto giuridico, in modo che il primo si manifesta come condizione (fattuale o immateriale ${ }^{42}$ ) del secondo. A tale categoria possono essere ricondotte sia le norme dotate di una struttura ipotetica ("Se F allora C"), sia quelle che «non paiono riconducibili alla forma standard di un enunciato condizionale, quali ad esempio: le norme definitore, le norme abrogatrici, le norme di interpretazione autentica» ${ }^{43}$. Spesso, come si è notato, l'effetto giuridico (il dover essere) che esse prescrivono contribuisce a formare la protasi, ossia I'antecedente logico, di altre regole (anche di comportamento) di carattere ipotetico ${ }^{44}$.

In secondo luogo, di notevole importanza sono le "regole attributive di competenza", ossia quelle che ci informano, rispetto ad una determinata "fonte-atto normativo", "chi" sia tenuto alla sua emanazione. Non solo: I'autorità normativa indicata, normalmente, è legittimata a produrre quel determinato tipo di fonte in via esclusiva, così, a fianco all'attribuzione di competenza, tali regole, da un lato, vietano ad altre autorità normative di produrre quel tipo di "fonte-atto", dall'altro, forniscono un criterio di identificazione necessario (ma non sufficiente) della fonte in questione ${ }^{45}$. Questo tipo di norme si differenzia sia dalle regole di comportamento, sia dalle regole costitutive: dalle prime in quanto l'attribuzione di un potere non implica di per sé alcun obbligo relativo al suo esercizio, dalle altre per il fatto che «attribuire un potere non è ricollegare un effetto (giuridico) ad un fatto, ma semmai stabilire chi ha il potere di produrre l'effetto» ${ }^{4}$.

Anche questo tipo di regole, come quelle costitutive, tendono a collegarsi con altri enunciati, contribuendo alla formazione di regole di comportamento. Si è già detto, tuttavia, che, in relazione al 42 Vedi G.U. RESCIGNO. L'atto normativo, cit., p. 39: «Il fatto (che la regola dice essere produttivo di quello specifico effetto con il suo solo accadere) può essere un fatto fisico-naturale [...] oppure un atto cioè qualcosa in quanto voluto da un soggetto[...]».

43 Vedi R. GUASTINI. Le fonti del diritto, cit., p. 22.

44 Ad esempio: "il soggetto A se è tutore legale di un soggetto minore allora è obbligato a provvedere all'educazione"; "il comportamento A se c'è abrogazione della norma che lo vieta allora è divenuto lecito"; "Se la norma x esprime il contenuto y allora y è il suo significato", ecc...

45 Cfr. R. GUASTINI. Le fonti del diritto, cit., p. 28.

46 Vedi G.U. RESCIGNO. L'atto normativo, cit., p. 42. 
contesto normativo, dalla sola attribuzione di una determinata competenza normativa ad un organo specifico si può ricavare in via implicita il divieto per gli altri organi di emanare quel tipo di fonte e, dunque, una "norma giuridica in senso stretto" (ossia una regola di comportamento).

Ancor più spesso, però, le regole sulla competenza sono accompagnate da enunciati che esprimono "modalità deontiche" relative all'esercizio del potere normativo, al suo oggetto e, talvolta, anche al modo in cui quest'ultimo deve essere disciplinato. Tali enunciati formano quelle che vengono definite dalla dottrina "norme sulla produzione", tra le quali si distinguono, inoltre, quelle che riguardano il dato "formale" e quelle che riguardano l'aspetto "materiale" delle fonti cui si rivolgono. Sotto il primo profilo, devono essere ricondotte le norme attributive di competenza e quelle che disciplinano il procedimento di formazione della fonte-atto. Sotto il secondo, invece, sono comprese le norme che circoscrivono o riservano l'oggetto di disciplina e quelle che limitano "direttamente" (attraverso comandi e divieti) o "indirettamente" (circoscrivendo le opzioni possibili al "legislatore") il contenuto che può assumere la disciplina dettata da una fonte ${ }^{47}$.

Le regole sulla produzione giuridica non si contrappongono alle altre per la loro struttura sintattica ma in quanto assumono la qualità di «"norme sulle norme" o "norme di secondo grado", dal momento che esse regolano appunto la formazione, l'interpretazione, ecc. delle disposizioni e delle norme che costituiscono I'ordinamento giuridico» 48 .

Le regole costitutive e quelle attributive di competenza, dunque, non prescrivono alcun comportamento: il loro contenuto sussiste e si realizza senza che se ne richieda una concreta esecuzione, proprio perché si colloca su un piano ideale e non materiale. Diversamente, le regole sulla produzione "vengono o non vengono eseguite": tuttavia, proprio per la loro funzione chiaramente strumentale rispetto alla formazione delle regole di comportamento "in senso stretto", vengono distinte da queste ultime e definite "norme secondarie". La funzione di queste tre tipologie di regole, dunque, è quella di dare forma e struttura sia al sistema sia al discorso giuridico, costruendo i "passaggi" necessari all'individuazione della norma giuridica (generale ed astratta).

La realtà materiale e le domande di giustizia espresse dal corpo sociale, costituiscono il terreno di prova della "progettazione giuridica" nel suo complesso. Gli enunciati sulla produzione normativa e quelli costitutivi dunque vengono collegati alle regole di comportamento in base alla ricostruzione che viene compiuta di volta in volta dall'operatore giuridico. Pertanto, si può osservare che «tutte le norme in senso generico sono funzionalmente dipendenti dalle prescrizioni» ${ }^{49}$. Nondimeno, benché costituiscano il "fulcro della produzione normativa", le regole di comportamento astratte e generali, trovando il loro fondamento nelle norme sulla produzione giuridica, sono strutturalmente e gerarchicamente subordinate a queste ultime.

Occorre distinguere, infatti, tra quella supremazia (gerarchica) che deriva «dalla diversa forza politica che sta alla base delle manifestazioni di volontà dello Stato (Costituzione, legge, regolamento, ecc. ) ${ }^{50}$, e quella che invece è «fondata su un rapporto di strumentalità logica che esiste tra le norme sulla produzione giuridica e le norme prodotte» ${ }^{51}$.

La struttura dell'ordinamento giuridico dunque può essere ricostruita in maniera differente a seconda che si abbia riguardo all"efficacia giuridica"52 della fonte nel momento storico in cui è emanata o, piuttosto, si voglia giustificare "I'effettivo funzionamento" del sistema normativo. Lasciamo ad un momento successivo i problemi relativi alla gerarchia materiale e ci occupiamo del secondo tipo di rapporto tra le norme, sulla base del quale abbiamo finora distinto i differenti tipi di regole (di comportamento, costitutive, di competenza) e i differenti contenuti deontici che esse

47 Per esigenze di sintesi, si preferisce schematizzare così le classificazioni della dottrina giuridica. Per un approfondimento ulteriore, cfr. R. GUASTINI. Le fonti del diritto, cit., p. 27-33.

48 Vedi A. PIZZORUSSO, Corso di diritto comparato, cit., p. 18. Dello stesso A., cfr. Delle fonti del diritto, in SCIALOJA e BRANCA (a cura di), Commentario al codice civile, Bologna-Roma, 1977, p. 6.

49 Vedi R. GUASTINI, Le fonti del diritto, cit., p. 20-22. Analoga ricostruzione è compiuta da G.U. RESCIGNO, L'atto normativo, cit., p. 46-47.

50 Vedi A. PIZZORUSSO. Delle fonti del diritto, cit., p. 12.

51 IVi, p. 11.

52 Il termine "efficacia" in riferimento ad una norma giuridica può essere utilizzato per indicare tre aspetti differenti: la sua capacità di produrre effetti giuridici; la sua "applicabilità" ad un caso concreto; la sua "forza", cioè la sua collocazione nella gerarchia dell'ordinamento, cfr. R. GUASTINI. Le fonti del diritto, cit., p. 258-259. Qui il termine è usato nell'ultimo dei sensi indicati. 
possono assumere (norme primarie e norme secondarie). Vi è da dire che in molti casi le norme sulla produzione, sono munite di una superiorità, oltre che "logica", anche "gerarchica" rispetto alle fonti di produzione a cui si riferiscono: così, ad esempio, sono la Costituzione e i regolamenti parlamentari che disciplinano il procedimento di formazione delle leggi, mentre, a loro volta i regolamenti governativi trovano la loro disciplina in atti legislativi (come in Italia, nella L.400/1988). Tuttavia, la "gerarchia basata sul carattere strumentale delle norme" non necessariamente opera in parallelo a quella "basata sull'efficacia delle norme" stesse ${ }^{53}$. Invero, il diritto in quanto discorso presuppone necessariamente una sua logica formale, così come si è visto che la norma giuridica in quanto progetto di comportamento ne presuppone un'altra di contenuto più ampio che la comprenda e le dia fondamento. Il fatto che poi quest'ultima venga espressa da fonti dotate di "forza" maggiore secondo la gerarchia materiale, è un dato riscontrabile negli ordinamenti dotati di una Costituzione "rigida", mentre in quelli aventi Costituzione "flessibile" la gerarchia rimane soltanto sul piano "strutturale" o "formale"54. In ogni sistema giuridico, si possono rintracciare, pertanto, norme secondarie, a prescindere dal rango da esse concretamente assunto ${ }^{55}$.

\section{L'EFFETTIVITÀ E LA VALIDITÀ DEL DISCORSO GIURIDICO.}

L'esistenza di una norma implica la sua appartenenza ad un qualche ordinamento giuridico e generalmente troverà fondamento sulle "norme secondarie" di quest'ultimo, giacché ciascun ordinamento ne possiede. Tuttavia, questa ricostruzione non basta a dare spiegazione di due questioni. In primo luogo, anche ammesso che vi sono delle norme sulla produzione che, a loro volta, trovano fondamento in altre norme sulla produzione ${ }^{56}$ (di rango pari o superiore), è chiaro che, tuttavia, non si può procedere in un regresso all'infinito: dunque, devono necessariamente esistere delle norme "auto fondate". In secondo luogo, il rapporto tra norme "primarie" e "secondarie" non è un rapporto "necessario", in quanto è dato riscontrare delle regole di comportamento che non trovano fondamento sulle norme sulla produzione e vengono pertanto chiamate "extra ordinem".

Il primo dei due rilievi conferma che nessun sistema giuridico può trovare la spiegazione della sua esistenza in sé stesso e che, invece, necessariamente la sua fondazione risiede nei "fatti sociali", ossia in fatti e attività dei membri di una determinata comunità. Il secondo dimostra che, talvolta, la produzione di norme non avviene all'interno della logica del sistema giuridico, ma attraverso un percorso "pratico". Tutto ciò malgrado le fonti extra ordinem costituiscano, come è ovvio, un'anomalia all'interno dell'esperienza giuridica dal momento che la loro esistenza deroga, anzitutto, l'indispensabile esigenza di "certezza" del diritto ${ }^{57}$, senza la quale non ha senso "progettare comportamenti futuri" attraverso enunciati prescrittivi.

La complessità della fenomenologia giuridica, dunque, non si può sciogliere soltanto attraverso la logica del linguaggio normativo, ma richiede di fuoriuscire dal sistema e di considerare le norme in base alla loro "effettività", ossia alla materiale accettazione ed osservanza da parte della generalità dei consociati.

Il "principio di effettività", tuttavia, assume un diverso connotato a seconda che venga chiamato a dare giustificazione alle fonti sulla produzione ( $\mathrm{e}$, di conseguenza, all'intero ordinamento) oppure a norme singole (le fonti extra ordinem).

53 Anzi, vi è un unico caso in cui ciò avviene necessariamente: «è quello delle norme istitutive di singole fonti [...] le quali non possono assegnare agli atti normativi o alle norme derivanti dalle fonti che istituiscono, un efficacia superiore alla propria». Vedi A. PIZZORUSSO, Delle fonti del diritto, cit., p. 9.

54 Si distingue pertanto tra una relazione di gerarchia "formale", ossia quando il rapporto di dipendenza si manifesta solo sul piano (strutturale) della "costruzione" del sistema giuridico e una relazione di gerarchia "materiale" o "contenutistica" in base alla quale le norme di rango inferiore non possono assumere contenuto contrastante con quelle di rango superiore. Cfr. R. GUASTINI. Il diritto come linguaggio, cit., p. 96.

55 Cfr. C. ESPOSITO, Validità delle leggi. Studi sui limiti della potestà legislativa, i vizi degli atti legislativi e il controllo giurisdizionale, Padova, 1934, 59 e ss.

56 Ad esempio, la L. 400/1988 che disciplina la potestà regolamentare del Governo, in quanto fonte primaria,trova fondamento sulle norme della Costituzione che disciplinano il procedimento legislativo (art.70 e ss.). Allo stesso modo, fino alla riforma costituzionale del 2001, non esistendo alcun riferimento costituzionale alle fonti comunitarie derivate (regolamenti e direttive), si riteneva che queste trovassero il loro fondamento nella legge di ratifica dei Trattati. In questo modo però si smentiva chiaramente il principio per cui nessuna fonte può istituirne un'altra di rango superiore.

57 Cfr A. PIZZORUSSO. Delle fonti del diritto, cit., p. 21. 
Nel primo caso, il principio di effettività viene in rilievo quale «base razionale, di tutto il sistema di produzione del diritto [...] cioè come la norma fondamentale del sistema delle norme sulle fonti, che ha il suo grado successivo nel complesso delle norme sulla produzione giuridica ed il terzo nelle norme prodotte»58.

Diversamente, con riferimento ad una singola norma, sulla base del principio di effettività si può avere il caso in cui essa «deve considerarsi giuridicamente esistente anche se non risponde ai criteri di validità dell'ordinamento vigente» in quanto generalmente accettata ed osservata dai destinatari; oppure, «non può considerarsi giuridicamente esistente anche se risponde ai criteri di validità dell'ordinamento vigente qualora non sia per nulla accettata ed osservata dai suoi destinatari» ${ }^{59}$. Nella prima eventualità saremmo di fronte ad una fonte extra ordinem, nella seconda, ad una "semplice" violazione ${ }^{60}$ : ciò che importa, tuttavia, è che sia con riferimento al sistema giuridico sia con riferimento alla regola singola, non si è potuto dare spiegazione del "concreto operare" del diritto oggettivo se non attraverso il principio di effettività e, pertanto, al di fuori di qualsiasi pretesa di coerenza logica rispetto l'insieme delle norme sulla produzione.

Esiste, pertanto, un limite al "discorso giuridico" dal momento in cui si può riscontrare un fondamento extralegale, non solo per l'intero ordinamento, bensì anche per la singola norma. I principio di effettività non si ricava da norme giuridiche, bensì dai fatti 0 , come è stato detto ${ }^{61}$, dalla "necessità": esso nulla ci dice rispetto alla norma a cui si riferisce, se non che "opera effettivamente", ossia viene accettata ed osservata dai suoi destinatari. Il "progetto" disegnato dalle norme sulla produzione viene così interrotto e il discorso giuridico ricomincia da premesse differenti e non conformi con quelle delle restanti fonti del diritto che invece seguono la gerarchia formale e materiale vigente nell'ordinamento giuridico.

Mediante il principio di effettività, dunque, la produzione normativa prende piede a partire dalla "necessità" (intesa in senso lato), la quale si sostituisce ai criteri di validità formali e materiali affermando, ex post, l'idoneità di comportamenti uniformi e continui nel tempo (osservanza della regola), uniti alla convinzione di obbedire ad una norma giuridica (opinio iuris seu necessitatis), ad essere fonti di produzione del diritto. Proprio con riferimento al momento temporale in cui avviene il "riconoscimento" della norma rispetto alla normalità dei casi (ex post), si dovrebbe parlare di «effettività legittimante», la quale non potrà che manifestarsi «nel reale conformarsi dell'esperienza alle norme, nel concreto prodursi degli effetti da questi disposti» ${ }^{62}$.

Nei casi riportati, la circolazione del diritto, ossia la sua "comunicazione sociale", non è più deducibile dal progetto disegnato dalle disposizioni normative, pertanto, il discorso diviene "incerto" dal punto di vista della logica giuridica: è evidente che nel caso delle norme che danno "fondazione all'ordinamento" e delle fonti extra ordinem non esiste alcun "rapporto di derivazione".

A tal proposito, questo complesso di norme e disposizioni storicamente determinate sono state definite "fonti originarie" «la cui appartenenza all'ordinamento non dipende dalla (previa) esistenza di altre norme ${ }^{63}$. La loro creazione, dunque, è avvenuta in assenza di limiti e di pretese di correttezza rispetto ad una qualsiasi altra norma: esse costituiscono per questo motivo il frutto di un potere costituente, il quale non può che trovare fondamento nella "materiale necessità" manifestatasi nel corso della storia.

Per una completa conoscenza della fenomenologia giuridica, dunque, occorre chiarire che cosa intendiamo quando all'interno della dottrina si è fatto e si fa riferimento al concetto di

58 Ivi, p. 11-12.

59 Vedi R. GUASTINI. Le fonti del diritto, cit., p. 75.

60 Secondo A. PIZZORUSSO. Corso di diritto comparato, cit., p. 7, tuttavia, «il fatto che una norma sia violata molto spesso, o addirittura in tutti i casi noti, non basta a far ritenere che essa sia priva di effettività, quando non sia venuto meno il generale o prevalente convincimento della sua capacità di operare come norma giuridica».

61 Cfr. A. PIZZORUSSO. Delle fonti del diritto, cit., p. 11; cfr. V. CRISAFULLI. Lezioni di diritto costituzionale, II, Padova, 1993, p. 193 e ss.; cfr. F. MODUGNO. Appunti dalle lezioni sulle fonti del diritto, Torino, 2005, p. 168 e ss.

62 Vedi V. CRISAFULLI. Lezioni di diritto costituzionale, cit., p. 194. L'A. sottolinea l'analogia tra i presupposti delle fonti extra ordinem e quelli della consuetudine. La differenza, tuttavia, si verificherebbe nel fatto che nel primo caso, la consuetudine sarebbe "confermativa" delle norme già poste piuttosto che costitutiva.

63 Vedi R. GUASTINI. Le fonti del diritto, cit., p. 61. 
"necessità". Sarebbe vano cercare di intendere la necessità come un processo a cui è riconosciuta I'idoneità a produrre la nascita, la modificazione o l'estinzione di norme giuridiche ${ }^{64}$ : la relazione di "riconoscimento" richiederebbe l'esistenza di un "qualcos'altro" (una regola) che indichi la necessità come fonte del diritto. Si riproporrebbe in tal modo, lo stesso dilemma da cui si è partiti, ossia trovare una legittimazione giuridica alla norma di riconoscimento della necessità. Si dovrebbe invece fuoriuscire dal linguaggio prescrittivo, lasciandoci indirizzare dal dato realmente percepito: «il concetto di "necessitas ius constituit" esprime, in forma sintetica, un massima di esperienza, come la proposizione "la volontà di Dio è fonte del diritto" esprime una massima di religione, ma né I'una né l'altra sono suscettibili di ricevere il valore di canoni giuridici» ${ }^{65}$. Tuttavia, pur non essendo una fonte del diritto, la necessità «quando si traduce in effettività, opera come presupposto del funzionamento 0 anche della creazione di una o più norme giuridiche» ${ }^{66}$.

Alla luce di quanto detto, mentre le "fonti derivate" appartengono ad un determinato ordinamento in quanto siano conformi alle norme sulla produzione giuridica $(0$, in altre parole, "in quanto siano valide"), le "fonti originarie" trovano il loro criterio di riconoscimento non in una norma "interna", bensì in una norma di diritto internazionale consuetudinario67, qual è il principio di effettività. Da ciò deriva che le fonti originarie si possono identificare, da un lato, in quei fatti che danno vita alla "prima costituzione"68, dall'altro, in quelle che abbiamo chiamato fonti extra ordinem.

Tuttavia, occorre chiedersi se il principio di effettività operi soltanto in relazione a questa tipologia di fonti che, proprio per l'elemento di "novità" che immettono nell'ordinamento abbiamo definito "originarie", o piuttosto sia possibile rintracciare una sua influenza anche verso le altre norme.

Anzitutto, trattandosi queste ultime di "fonti derivate", si presuppone che comunque ci sia stato un atto di creazione da parte di un'autorità normativa. Cionondimeno, come è stato notato, "I'atto di creazione" può dar luogo ad "esistenza" della norma giuridica al di fuori dei "limiti" segnati dal concetto di validità. Si è accennato sopra al fatto che il concetto di "validità" e quello di "esistenza" esprimono entrambi una "qualità relazionale", ma, mentre la "validità" costruisce una relazione con altre norme, I"esistenza" descrive una relazione di appartenenza ad un ordinamento. In tal modo, si può osservare che «una norma esiste quando sia stata emanata de facto da un organo in prima facie competente» ${ }^{69}$. L'esistenza della norma giuridica, dunque, "richiede assai meno" della sua validità, sebbene essa possa essere sovrapposta, in linea di massima, alle condizioni di validità formale ${ }^{70}$. Rimane, infatti, un margine di scarto tra le due qualità: un margine che, ragionando con un percorso ipotetico, ci permette di individuare di nuovo nel principio di effettività il fondamento ultimo della giuridicità, o meglio della prescrittività di un enunciato. Sulla base delle premesse appena esposte, nel caso in cui un atto normativo sia stato prodotto da un organo palesemente incompetente o vi siano state evidenti violazioni delle "norme sulla procedura di produzione" nel suo processo di creazione, questo dovrebbe dirsi "inesistente". Si ponga, tuttavia, l'ipotesi, sicuramente eccezionale, in cui, per un qualsiasi motivo, l'invalidità dell'atto non venga rilevata dall'organo competente e il suo contenuto venga comunque osservato ed accettato dalla generalità dei cittadini: in questo caso, esattamente come per le fonti extra ordinem, saremmo di nuovo davanti ad un caso di riconoscimento "ex post" della norma. Si è parlato, pertanto, di «effettività legittimante» proprio con riferimento alla capacità che ha il principio in questione di "rovesciare" i tempi della produzione giuridica dominati dal normale rapporto di sequenza tra le norme sulla produzione e quelle di comportamento. Il fatto normativo 64 Cfr. T. PERASSI, Necessità e stato di necessità nella teoria dommatica della produzione giuridica (1917), in Scritti giuridici, I, Milano, 1958, p. 195.

65 IVi, p. 199.

66 Vedi A. PIZZORUSSO. Delle fonti del diritto, cit., p. 11.

67 Cfr. R. GUASTINI. Le fonti del diritto, cit., p. 64.

68 Secondo R. GUASTINI. Le fonti del diritto, cit., p. 63, «[...] al vertice della piramide non necessariamente vi è una singola norma suprema: possono esservene molteplici, tutte pari-ordinate, perché formulate in un unico documento normativo, prodotto da un potere di fatto. Il documento che esprime I'insieme delle supreme norme di un ordinamento è appunto la sua (prima) costituzione».

69 Ivi, p. 267.

70 Ivi, p. 258. L'A., comunque, precisa che non può essere stabilito a priori quali norme sulla produzione sia necessario osservare nel procedimento di creazione dalla norma giuridica, affinché quest'ultima possa dirsi esistente. È chiaro che una palese incompetenza di un organo ad emanare una norma giuridica, così come la palese violazione della procedura, porterebbe ad escludere anche l'esistenza stessa della norma, la quale, nell'ipotesi limite in cui goda comunque di generale osservanza, potrebbe al massimo essere qualificata come fonte extra ordinem. 
fondato sul principio di effettività è stato definito "normogeno bivalente" poiché esprime, in due differenti momenti, sia la norma prodotta che la norma sulla produzione: quest'ultima, infatti, «è espressa a posteriori», ossia «dallo stesso fatto normativo all'atto del suo affermarsi» ${ }^{71}$.

Al di fuori di queste ipotesi, pur importanti ai fini della spiegazione dell'esperienza giuridica nel suo complesso, tra norme sulla produzione e norme di produzione si instaurano due tipi di relazione: da un lato, esiste una pretesa di corrispondenza in quanto si richiede che la norma sia stata creata dall'organo competente e secondo il procedimento prescritto (validità formale); dall'altro, una pretesa di non contraddizione poiché la norma prodotta non può avere un contenuto incompatibile con le norme, materialmente sovraordinate, che ne limitano o prestabiliscono il contenuto (validità materiale). Tuttavia, come si è visto, validità ed esistenza esprimono due concetti indipendenti: possono benissimo darsi, infatti, delle norme invalide all'interno dell'ordinamento giuridico (quali ad esempio, leggi costituzionalmente illegittime o regolamenti contrari alla legge) le quali continuano ad operare ed a produrre effetti finché un giudice non ne rilevi i vizi e ne disponga I'annullamento. Per questa ragione, qualora per "norma" si intenda un enunciato di carattere prescrittivo (comando o divieto) dinnanzi alla quale, a prescindere dal contenuto che assume, ci si attende una "conformazione" da parte dei consociati, dovremmo concludere, come si è già detto, che «dal punto di vista teorico l'unico criterio valido per misurare la giuridicità di una norma è quello dell'effettività dell'influenza da essa esercitata» ${ }^{72}$, ossia il fatto che sia generalmente accettata ed osservata all'interno di una determinata collettività.

La validità, quale "criterio di riconoscimento", esprime un giudizio descrittivo, poiché definisce le condizioni in presenza delle quali un atto, una disposizione o una regola "possano dirsi" coerenti con I'ordinamento; I'efficacia vincolante, ossia la giuridicità, trova invece nel principio di effettività il suo fondamento. Di conseguenza, «i giudizi di validità sono enunciati "esterni": enunciati dunque del discorso descrittivo ${ }^{73}$.

L'obbligatorietà di un certo comportamento, invece, può derivare solo da un enunciato prescrittivo, in questo caso, da una meta-norma, regola di comportamento, che prescriva l'obbedienza ad un'altra regola di comportamento. Solo in questo modo può ritenersi esistente un obbligo (giuridico e non semplicemente "morale") di applicazione ${ }^{74}$. L'obbligatorietà (o applicabilità) costituisce, pertanto, «un concetto descrittivo (di una relazione triadica: tra due norme e un caso) e, inoltre, relativo (a un ordinamento giuridico e a determinati casi) $\gg^{75}$.

In tale contesto, risulta fondamentale, come vedremo, il ruolo delle autorità (giurisdizionale e non) per la quale il "riconoscimento" non sarà sempre agevole. Al contrario, «quanto meno precisi, differenziati, univoci sono i criteri positivamente adottati per caratterizzare fonti e norme di diritto oggettivo, tanto maggiore è il ruolo del giudice (nel senso più largo di organo chiamato ad applicare autoritativamente e super partes il diritto oggettivo) $\gg^{76}$.

\section{L'UNITÀ DELL'ORDINAMENTO E L'APPLICAZIONE DELLE DISPOSIZIONI.}

Esattamente come il discorso giuridico si struttura attorno ad un determinato caso per mezzo di una pluralità di enunciati la cui concordanza logica condiziona la validità della norma individuale conclusiva, allo stesso modo, il sistema giuridico è composto da una pluralità di fonti che, data "I'inesauribilità della produzione giuridica", lo aggiornano continuamente in base alle necessità storicamente determinatesi.

71 Cfr. F. MODUGNO. Appunti dalle lezioni sulle fonti del diritto, cit., p. 167.

72 Vedi A. PIZZORUSSO. Corso di diritto comparato, cit., p. 7.

73 Vedi R. GUASTINI. Le fonti del diritto, cit., p. 273. Gli enunciati "esterni" si possono formulare nella qualità di osservatori della fenomenologia giuridica. Differentemente gli enunciati "interni" implicano un'accettazione etico-politica delle norme di condotta. Il concetto di validità per I'A., non appartiene al linguaggio normativo, bensì a quello della dogmatica giuridica, la quale, come "teoria generale", «non si interroga sul contenuto dell'ordinamento, ma sul modo migliore di descriverlo quale che sia», Ivi p. 56.

74 Cfr. BULYGIN. Il positivismo giuridico, cit., p. 61.

75 Ibidem.

76 Vedi V. CRISAFULLI. Lezioni di diritto costituzionale, II, cit., p. 186. 
Pertanto, le disposizioni incessantemente prodotte dalle fonti (se non altro in quanto rispondono a differenti "domande sociali"), possono entrare tra di loro in antinomia.

Il concetto di validità, in questo senso, può essere visto come un prodotto della dogmatica giuridica: il postulato dell'unità del sistema ${ }^{77}$ si realizza per mezzo della gerarchia strutturale e materiale, nonché, prima ancora, per mezzo del criterio "cronologico" lex posterior derogat priori.

Abbiamo parlato finora del concetto di validità con riferimento alle "norme giuridiche" in generale, accennando al fatto che esso può riguardare sia il profilo strutturale sia il contenuto della norma.

A tal proposito si parla di validità formale, per indicare il rispetto delle norme sulla produzione poste alla base della gerarchia strutturale (dinamica) dell'ordinamento, ossia quelle che individuano l'organo legittimato in relazione ad determinata fonte di produzione (regole attributive di competenza) e quelle che disciplinano il procedimento di esercizio del potere normativo. Le norme sulla produzione in senso stretto, dunque, non "dicono" nulla rispetto al contenuto, ossia in merito al significato che dovrebbe esprimere l'atto linguistico espresso da determinata fonte di diritto. L'oggetto del giudizio di validità formale è infatti I'atto normativo, ossia il contenitore della norma, anche se è logico che poi, l'eventuale dichiarazione di invalidità dell'atto, impedisca di produrre qualsiasi effetto anche al suo contenuto, ossia ad ogni singola disposizione e alle regole che da ciascuna di esse possono essere ricavate.

Il concetto di validità materiale, invece, guarda direttamente al "significato", ossia alle norme in senso stretto, e le collega alle fonti sulla produzione attraverso una relazione di non incompatibilità. In tal senso, la validità materiale costituisce il criterio di ricostruzione della progettualità connessa ad un determinato sistema di regole e postula che ogni "scala" del progetto deve essere a sua volta "prevista e contenuta" in una superiore. La pretesa di coerenza che avanza deve necessariamente essere rapportata alle caratteristiche semantiche della disposizione e alle scelte operate dall'interprete circa la regola da applicare al caso concreto ${ }^{78}$.

L'obbiettivo dell'unità dell'ordinamento giuridico, in questo senso, non può che essere rimandato al momento dell'applicazione, per due ragioni. In primo luogo, poiché così residueranno solo le "antinomie materiali", ossia le contraddizioni tra norme che non possano essere ordinate secondo il criterio cronologico o di competenza (territoriale o materiale). In secondo luogo perché, posto che il principio di certezza richiede che la norma concreta ed individuale possa essere soltanto una, non è men vero che «si danno effettivamente [...] diverse e discordanti ricostruzioni dell'unità dell'ordinamento, quindi diverse possibili soluzioni delle antinomie che in questo si manifestino, in funzione del diverso significato attribuibile alle varie norme ${ }^{79}$.

L'interpretazione costituisce quindi il principale terreno di prova delle varie ricostruzioni del sistema giuridico, le quali necessariamente influiscono sulla individuazione della norma relativa al caso concreto. Ovviamente la questione assume un'importanza fondamentale con riferimento alla validità materiale, dal momento che ogni disposizione può esprimere alternativamente o congiuntamente più "significati", ossia più norme, delle quali può darsi che solo uno o alcuni abbiano un contenuto non contrastante con le norme sovraordinate. In questo senso, si è inteso sostenere che il postulato dell'unità dell'ordinamento giuridico trova la sua sede privilegiata nel momento dell'applicazione, in particolare negli organi più alti dell'apparato giurisdizionale, le cui decisioni sono vincolanti o persuasive per le corti inferiori.

È evidente a tal proposito la centralità assunta dalle "sentenze interpretative" (di accoglimento e di rigetto) dei tribunali costituzionali, i quali, pronunciandosi sui possibili significati di una disposizione, possono dichiarare l'invalidità di una o più norme, facendo, tuttavia, "salva" la disposizione nel suo insieme. Soltanto quando nessuno dei significati ricavabili dall'enunciato linguistico non sia contrastante con le norme materialmente sovraordinate, l'invalidità colpirà l'intera disposizione ${ }^{80}$.

77 Ivi, p. 206.

$78 I v i$, p. 208: «[...] la quale, per un principio di certezza implicitamente accolto dal nostro ordinamento, deve non poter essere, di volta in volta, che una sola».

79 Ivi, p. 209.

80 In questo senso si è espressa la Corte Costituzionale (sent. 448/2007), affermando che «nessuna disposizione di legge può essere dichiarata costituzionalmente illegittima solo perché suscettibile di essere interpretata in contrasto con i precetti costituzionali, ma dev'esserlo soltanto quando non sia possibile attribuirle un significato che la rende conforme alla Costituzione». 
Il rapporto tra le fonti collocate nelle diverse scale della gerarchia può essere compreso anche con riguardo all'attributo della generalità e dell'astrattezza di cui si è parlato a proposito dei diversi livelli del discorso giuridico. Ogni progetto, dovendo prevedere e de-limitare i progetti di scala minore, presenterà un certo livello di generalità ed astrattezza che si abbasserà scendendo la gerarchia delle fonti, mentre, al contrario, si farà sempre più elevato risalendola, fino al progetto "apicale" ed "originario" espresso della Costituzione ${ }^{81}$.

\section{L'INTERPRETAZIONE E IL LINGUAGGIO NORMATIVO.}

Il momento dell'applicazione delle disposizioni normative, al pari di quello della "sistemazione" delle fonti, costituisce uno dei problemi principali dell'esperienza giuridica. Come si è detto in precedenza, la distanza tra la "previsione" normativa e la sua "attuazione" si presta ad essere colmata in maniere differenti a seconda della ricostruzione del quadro normativo della vicenda che effettua I'organo dell'applicazione.

Di fronte ad una controversia, infatti, il giudice non ha soltanto il dovere di individuare la regola di comportamento relativa al caso concreto, ma deve anche giustificare perché ritiene che quella sia la sola validamente applicabile. La motivazione della sentenza, in questo senso, dovrebbe ricostruire I'iter logico che ha portato alla definizione del "dispositivo", ossia della norma puntuale e concreta.

La sussunzione, tuttavia, rappresenta solo il momento di chiusura di un "percorso applicativo" che ha inizio con l'individuazione della norma (generale ed astratta) alla quale si crede di ricondurre il caso individuale. La ricerca delle disposizioni normative e la loro interpretazione è contraddistinta da una notevole quantità di variabili che intervengono nell'esito dell'operazione e che sono determinate dalla percezione del tessuto normativo maturata dall'interprete. Sembra difficile, tuttavia, concludere che quest'ultima, essendo il frutto di un procedimento intellettuale soggettivo, valga solo nei confronti dell'interprete e pertanto non possa estendere la sua validità oltre la mente di quest'ultimo. Al contrario, è naturale per gli uomini scambiare le conoscenze in loro possesso attorno ad un determinato fatto, specie se di comune interesse ${ }^{82}$ : in questa maniera, all'interno del discorso, le percezioni individuali vengono rielaborate e perfezionate fino al momento in cui i partecipanti non raggiungono un consenso circa un determinato tipo di rappresentazione dell'oggetto di indagine. La descrizione, ossia il complesso di enunciati che esprime la percezione, acquista un valore "intersoggettivo" in quanto "accettata" dagli altri partecipanti al dialogo ${ }^{83}$. Dati questi presupposti, si potrà argomentare che la maggiore o minore estensione del "consenso" attorno ad una determinata "comprensione" circa l'oggetto del discorso è definita dalla maggiore o minore "correttezza" degli atti linguistici che la descrivono. Ciò vale per tutti i tipi di dialogo: com'è stato autorevolmente rilevato, anche in ambito scientifico la fondatezza dei risultati di una ricerca richiede la razionalità del linguaggio con la quale essa viene comunicata che si verifica qualora «le [...] preposizioni costituiscano un sistema coerente di enunciati» ${ }^{84}$.

Nel corso del novecento, ogni materia della ricerca scientifica ha sviluppato un proprio "linguaggio" individuato dalle regole che disciplinano, da un lato, I'utilizzo dei concetti espressi dalle singole 81 «Quanto più si scende, perciò, nella gerarchia [materiale], tanto più numerosi e consistenti diventano i limiti che la capacità creativa di nuovo diritto delle fonti incontra nelle norme degli strati superiori (poste, cioè, da fonti dotate di maggior forza); mentre, all'inverso, i limiti vanno diradandosi man mano che si risale verso l'alto, fino a cessare del tutto nei confronti delle fonti supreme». Vedi V. CRISAFULLI. Lezioni di diritto costituzionale, $I I$, cit., p. 229.

82 Nel momento in cui inizia la comunicazione si sviluppa attraverso il "dialogo" una «produzione teorica multipla». Ciò avviene in quanto, «per cogliere i fenomeni che li circondano e li preoccupano, ma anche per consentire l'esistenza di una comunicazione sociale, gli uomini producono dei "discorsi"», vedi M. MIAILLE. Introduzione allo studio critico del diritto, cit., p. 23. A ciò si aggiunga, come si è chiarito e si provvederà ad approfondire, che, se intendono "comunicare comprendendosi", gli uomini non possono fare a meno di stabilire delle regole circa la "formazione" e la "riproduzione" dei loro discorsi.

83 Così, come osserva N. BOBBIO, Scienza del diritto e analisi del linguaggio, cit., p. 346, «la scienza ha inizio con la formazione del cosiddetto linguaggio scientifico, cioè con quel linguaggio che fa di una conoscenza puramente soggettiva (la mia percezione) una conoscenza al massimo grado intersoggettiva».

84 Ibidem 
parole degli enunciati (regole di formazione delle preposizioni iniziali), dall'altro, la "derivazione" di preposizioni a partire da altre "originarie" o "primitive" (regole di trasformazione delle preposizioni iniziali) ${ }^{85}$. Quanto più il discorso scientifico è rigoroso, ossia, quanto più I'utilizzo dei concetti e la trasformazione delle preposizioni originarie si attengono alle regole prestabilite, tanto più elevato sarà il valore intersoggettivo della "conoscenza" che esprime.

In epoca contemporanea, quindi, si assiste al passaggio «dalla concezione della scienza come apprendimento della verità alla concezione della scienza come sistemazione rigorosa dei concetti a fini pratici» ${ }^{86}$. In questo senso, il concetto scientifico non avanza una pretesa di oggettività, ma diviene "strumento di comunicazione" della conoscenza, la cui spendibilità nel discorso scientifico dipende dalla precisione osservata nel determinare le regole del suo utilizzo.

Venendo alla scienza giuridica, come si è detto finora, I'oggetto di studio del giurista sono le regole di comportamento, le quali, a partire dall'epoca illuminista, sono per la maggior parte formulate in preposizioni scritte, in opposizione alla tradizione medievale in cui, invece, vi era una prevalenza della fonte consuetudinaria. Il compito del giurista, pertanto, è quello di «trasformare il discorso legislativo in un discorso rigoroso ${ }^{87}$. Così mentre nelle scienze naturali, gli enunciati del discorso degli studiosi riguarderanno "fatti" ed "eventi" reali, nella scienza giuridica I'oggetto della descrizione saranno le "parole" dell'autorità normativa ${ }^{88}$. L'interpretazione pertanto, è l'insieme delle operazioni intellettuali che hanno come obbiettivo la specificazione del significato delle disposizioni normative, ossia, dal punto di vista pratico, il "medium" tra la comunicazione del legislatore e la comprensione da parte dell'autorità operante ${ }^{89}$. Il diritto oggettivo (ossia le norme ricavabili dalle disposizioni), è costituito da enunciati appartenenti al discorso prescrittivo (ossia né veri né falsi, ma soltanto eseguibili), mentre la dottrina giuridica, sviluppando un discorso scientifico, utilizza enunciati "descrittivi" (veri o falsi).

L'osservazione appena compiuta, tuttavia, determina l'emergere di una delle principali problematiche dell'interpretazione giuridica. Come abbiamo sostenuto fin qui, il "discorso giuridico" è formato dall'insieme degli enunciati (sulla produzione, sulla competenza, costitutivi, ecc..) che sono di volta in volta necessari a costruire la risposta (la regola di comportamento generale ed astratta) relativa ad un caso individuale ${ }^{90}$. Trattandosi di enunciati prescrittivi, le premesse e le conclusioni del ragionamento giuridico non possono essere oggetto di un'argomentazione di tipo logico-deduttiva.

Le preposizioni che compongono il ragionamento "conoscitivo", invece, sono vere o false ${ }^{91}$ : nel discorso delle scienze naturali e formali gli enunciati, in quanto ontologicamente diversi

85 Ivi, p. 345 e ss.

86 Ivi, p. 352.

87 Ivi, p. 350.

88 «Le scienze naturali sono discorsi che hanno ad oggetto fatti non linguistici o extra linguistici[...]. Il linguaggio della scienza [naturale] è ontologicamente eterogeneo rispetto al suo oggetto». La scienza giuridica, invece, «è un discorso che ha ad oggetto nulla più che un altro discorso».Vedi R. GUASTINI, Il diritto come linguaggio, cit., p. 211.

89 Cfr. G.U. RESCIGNO. Comunicare, comprendere interpretare nel diritto, cit., p. 694. Ai fini del ragionamento che si intende fare in questo paragrafo si ritiene sufficiente non approfondire ulteriormente circa il significato del termine "interpretazione". Vengono qui condivise, a tal proposito, le osservazioni dell'A., per il quale spesso si usa ricondurre sotto la parola interpretazione «cose troppo diverse» (ivi p. 722), mentre in realtà andrebbero almeno distinte quelle attività attraverso le quali, come vedremo, si "aggiungono frammenti di testo" alle preposizioni normative.

A tal proposito, è stato messo in luce che una prima importante distinzione và compiuta tra coloro che adottano un "concetto ristretto di interpretazione", per i quali essa avrebbe luogo solo nei casi in cui «il testo [risulta] oscuro o in una situazione di dubbio applicativo» e coloro che, invece, sostengono che I'interpretazione vi è sempre in quanto la decisione attorno al significato rappresenta un «presupposto necessario per l'applicazione», vedi R. GUASTINI. Il diritto come linguaggio, cit., p. 129. Il secondo orientamento coglie, in linea di principio, la contrapposizione tra l'elemento semantico e pragmatico, per cui qualsiasi attribuzione di significato venga compiuta nei confronti di un testo normativo, essa deve sempre trovare giustificazione alla luce della fattispecie concreta a cui si vuole applicare.

90 Cfr. G.U. RESCIGNO. L'atto normativo, cit., p. 44.

91 Nel senso in cui si è inteso finora, per "verità" dell'enunciato si intende che le parole di cui è formato siano utilizzate in conformità alle regole convenzionalmente fissate o, nel caso in cui l'enunciato in questione sia una "preposizione derivata", che siano state rispettate le "regole di trasformazione". 
dall'oggetto che studiano, non possono interferire con esso, ma soltanto descrivere le sue qualità. L'argomentazione quindi segue la logica deduttiva, pertanto, allorché gli enunciati che costituiscono le premesse sono veri, corrispondono a verità pure le conclusioni.

Stando così le cose, bisognerebbe negare ciò che si è affermato in apertura del capitolo, cioè la verificabilità del discorso giuridico secondo le regole della logica. Ma è proprio in questa sede, tuttavia, che diviene decisivo il ruolo della dottrina giuridica.

Se è vero che l'oggetto della scienza giuridica sono le regole di comportamento, invero, I'analisi del giurista non potrà non tener conto delle altre regole del linguaggio normativo (sulla produzione, costitutive, ecc...) che conferiscono struttura e collegano i vari enunciati del discorso giuridico. Le norme giuridiche "in senso generico", dunque, costituiscono la base principale per la formazione dei concetti e delle rappresentazioni che la scienza giuridica matura attorno all'ordinamento in generale ${ }^{92}$.

La costruzione di una logica del discorso giuridico, pertanto, passa attraverso l'analisi del linguaggio legislativo e, dunque, dalle regole che stabiliscono l'utilizzo delle parole espresse dagli enunciati dell'autorità normativa e delle regole di produzione delle preposizioni "derivate". Rispetto alle prime, occorre precisare che gran parte dei termini del discorso legislativo seguono le regole di utilizzo del linguaggio comune. Và altresì detto, tuttavia, che vi sono parole che richiamano concetti di una certa rilevanza per il linguaggio normativo o che non vengono usate secondo la prassi comune e, pertanto, si esige un maggior grado di rigorosità nella loro specificazione ${ }^{93}$. Quanto alle "regole di trasformazione", come si è visto, il diritto, esattamente come disciplina i comportamenti umani, regola la sua stessa creazione e riproduzione: le norme sulla produzione ed i criteri ordinatori delle fonti costituiranno pertanto i punti di riferimento attraverso i quali il giurista dà luogo a costruzioni teoriche relative alle qualità della norma come "validità/invalidità", "efficacia/inefficacia", "esistenza/ inesistenza", ecc... La spiegazione dell'ordinamento giuridico, dunque, coinvolge due livelli di discorso, quello del legislatore e quello della scienza giuridica.

Le premesse appena svolte ci permettono di tracciare ad una duplice conclusione, da un lato, circa la separazione tra il linguaggio legislativo e quello della scienza giuridica, dall'altro, rispetto alla possibilità di costruire ragionamenti logico-deduttivi, e quindi "cognitivi", con riferimento al linguaggio normativo.

Sotto il primo profilo, stando a quanto si è detto finora, il discorso del legislatore e quello della dottrina sono cose ben diverse. Cionondimeno, ci sembra opportuno notare che questa differenza sia destinata ad offuscarsi nel momento in cui si dà applicazione al primo. Nei ragionamenti giuridici gli enunciati che formano le premesse e le conclusioni del discorso non sono "norme", ma "proposizioni normative ${ }^{\prime 94}$, ossia enunciati che asseriscono determinate qualità delle norme. Un conto è «l'asserto che afferma che il diritto dispone una certa cosa, ed è una preposizione normativa, vera o falsa», un altro è l'asserto che prescrive o dispone alcunché, ossia la norma giuridica di cui si può dire solo se sia stata eseguita o meno ${ }^{95}$.

Dunque, venendo al secondo profilo, sebbene le regole di comportamento «siano prive di valori di verità, non sono però prive di valori di verità le preposizioni che vertono su di esse» ${ }^{96}$. Le regole della logica, pertanto, potranno applicarsi alle norme indirettamente, «per il tramite delle preposizioni

92 Senza contare, come vedremo, l'importanza che le tesi della dottrina hanno rispetto all'interpretazione dei testi normativi e, dunque, alla definizione delle regole di comportamento.

93 Si pensi a parole come «buon costume», «proprietà», «utilità sociale» o «finalità sociali», ecc... È da notare che spesso, in ambito dottrinale, si usa circoscrivere o specificare il loro "contenuto" mediante il collegamento con altri enunciati normativi, gerarchicamente o materialmente subordinati.

94 Cfr. GUASTINI, Il diritto come linguaggio, cit., p. 192. Sul punto, si veda anche E. BULYGIN, II positivismo giuridico, cit., p. 89 , il quale distinguendo tra norme e preposizioni normative, sostiene il carattere sostanzialmente descrittivo di queste ultime che, pertanto, potranno dirsi "normative" in quanto "relative a norme", ossia poiché «affermano che una determinata norma esiste, appartiene ad un determinato ordinamento, è applicabile ad un certo caso, o è efficace». L'A. sviluppa queste riflessioni sulla scorta della teoria di G.H. VON WRIGHT, Norma e azione. Un'analisi logica. Bologna, 1989; successivamente ripresa e sviluppata anche da C.E. ALCHOURRÒN. Logic of Norms and Logic of Normative Propositions, in Logique et analyse, 12, 1969.

95 Cfr. E. BULYGIN. Il positivismo giuridico, cit., p. 41.

96 Vedi R. GUASTINI. Il diritto come linguaggio, cit., p. 190 e ss. 
normative che le ripetono e le descrivono» ${ }^{97}$.

L'analisi del linguaggio si risolve nella formulazione di enunciati descrittivi, ossia in una produzione teorica in materia giurisprudenziale, la cui "fondatezza" è valutata alla luce delle regole del discorso scientifico giuridico. L'organo dell'applicazione, come vedremo, non potrà fare a meno di considerare le "preposizioni normative" e le tesi della scienza giuridica, sia nell'attività di ricerca delle norme sia nella sussunzione del caso concreto nelle fattispecie astratte delineate da quest'ultime. In questo senso, non sembra esatto definire "metà-linguaggio" quello della dottrina, giacché le conoscenze e le tesi (interpretative e dogmatiche) sviluppate dai giuristi divengono parte del ragionamento applicativo, ossia, linguaggio normativo.

Costruire un insieme di enunciati "coerente" e "unitario", pertanto, permette di ridurre i rischi di incomprensione tra l'autorità normativa e gli organi dell'applicazione, allo scopo di rendere meno vaghi i contorni del quid iuris.

Il significato delle disposizioni sarà ricavabile, da un lato, mediante gli usi linguistici comuni, le regole semantiche poste dall'autorità normativa98 e quelle sviluppate dalla scienza giuridica, dall'altro, dalla volontà e dalle finalità perseguite dall'autorità normativa nel produrre la disposizione ${ }^{99}$. Anche in quest'ultimo caso, tuttavia, l'interpretazione non fuoriesce dal contesto linguistico: qualsiasi indagine circa lo "spirito" di un determinato istituto o "I'intenzione del legislatore" dovrà necessariamente prendere le mosse dagli enunciati normativi in materia o, meglio, dalle "preposizioni normative" che ne descrivono il contenuto ${ }^{100}$. La "sistemazione dei concetti" svolta dalla dottrina giuridica adempie esattamente tale scopo. Il contenuto delle parole e, dunque, il "disegno generale" espresso dalla norma, dipende dal contesto proposizionale all'interno del quale sono inserite, nonché dalle norme linguistiche che vincolano il loro utilizzo. Dal momento che ciascuna proposizione non ha senso se non con riferimento ad altre, gerarchicamente o materialmente superiori o inferiori, che ne circoscrivono o specificano il contenuto, è innegabile che il suo valore cambierà in relazione alle "connessioni" che opererà il giurista con gli altri enunciati.

In conclusione, in linea teorica non sarebbe necessario fuoriuscire dal linguaggio dell'autorità ed immergersi in altri campi della scienza (la filosofia, la sociologia, l'etica, ecc...) per definire il significato delle parole che compongono una proposizione normativa: è evidente che il diritto conosce e "fa proprie" valutazioni del campo "morale" o di altre discipline scientifiche. Cionondimeno, il fatto che concetti extragiuridici siano incorporati nelle disposizioni normative, non evita la "trasformabilità" del significato che esprimono davanti all'aggiunta di nuovi riferimenti semantici da parte dell'autorità normativa o della scienza giuridica. Anzi, spesso sono gli stessi organi dell'applicazione che usano modificare i contesti linguistici al fine di conferire un significato nuovo alle disposizioni ed adattare il discorso normativo ai mutamenti della realtà ${ }^{101}$.

\section{L'ARGOMENTAZIONE E LA DECISIONE.}

Rimanendo sul percorso finora tracciato, è pacifico ritenere che il primo oggetto di indagine, per il giudice o per l'operatore giuridico tenuti a provvedere rispetto al caso concreto, sia I'atto linguistico (la disposizione) per mezzo del quale il legislatore ha inteso disporre un assetto normativo relativamente ad una tipologia di fattispecie astrattamente considerata.

Se si ipotizza un linguaggio legislativo unitario e coerente, la ricerca della disposizione da applicare non dovrebbe dar luogo ad esiti incerti ${ }^{102}$, pertanto, essa sarà rintracciabile seguendo le

97 Ivi, p. 192.

98 Ad esempio, l'art. 12 delle "preleggi" del codice civile.

99 Cfr. P. CHIASSONI, Presentazione, in E. BULYGIN, Il positivismo giuridico, cit., p. LX.

100 Sul punto si veda N. BOBBIO. Scienza giuridica e analisi del linguaggio. cit., p. 354-5, per il quale «il passaggio dall'interpretazione della lettera all'interpretazione dello spirito non rappresenta altro che l'estensione delle indagini sull'uso della parola in questione a contesti di preposizioni sempre più numerose e via via sempre meno affini al contesto iniziale».

101 Senza che ciò determini, come si è detto, che il linguaggio normativo si "colorisca" di preposizioni provenienti da altri rami delle scienze umane. Qui si vuole affermare che i mutamenti della realtà influiscono sulla scelta delle argomentazioni degli organi dell'applicazione, non che le premesse normative del ragionamento giuridico vadano interpretate facendo rinvio a preposizioni o ricerche di altri rami delle scienze.

$102 \mathrm{Cfr}$. N. BOBBIO. Scienza giuridica e analisi del linguaggio, cit., p. 350. L'A., tuttavia, illustra la 
"indicazioni" offerte dagli enunciati dell'autorità normativa.

Nella prassi giuridica, tuttavia, è possibile registrare due situazioni alternative: in alcuni casi, agli occhi dell'organo dell'applicazione, l'ordinamento "fornisce" in modo chiaro e inequivoco la regola di comportamento relativa al caso concreto, in altri, invece, permangono degli elementi di incertezza.

È stato osservato che, generalmente, questa seconda circostanza può verificarsi per tre ragioni: in primo luogo, può darsi che dalle disposizioni non possa ricavarsi alcuna norma che si riferisca al caso (lacuna normativa); in secondo luogo, è possibile che nell'ordinamento esistano due o più norme riconducibili alla fattispecie concreta, ma che esse manifestino soluzioni contrastanti (contraddizione normativa); infine, si riscontra una norma applicabile al caso concreto, ma la sussunzione risulta essere impraticabile a causa dell'indeterminatezza dei concetti utilizzati dal legislatore nel descrivere la fattispecie astratta (lacuna di riconoscimento) ${ }^{103}$.

In ognuna di queste ipotesi, I'intervento del giudice si risolve in una "innovazione" del diritto ${ }^{104}$ : nel caso in cui riscontri una lacuna normativa sarà costretto ad "aggiungere" una (nuova) norma relativa al caso specifico (ricavata per analogia da casi simili o da disposizioni di principio o altro ancora); nel caso di conflitto tra due norme il giudice dovrà "scegliere" di applicarne una escludendo le restanti; nell'ultima ipotesi, infine, il giudice, decidendo se ricondurre o meno la fattispecie concreta in quella astratta prevista in via legislativa, allarga o restringe la "descrizione" di quest'ultima.

La ricerca, in questi casi, comporta una vera e propria attività di "manipolazione": I'assenza di disposizioni normative, o comunque, l'equivocità del linguaggio del legislatore, richiede un'analisi "critica" dell'ordinamento giuridico che, ad ogni modo, non può che prendere le mosse dalle disposizioni normative stesse e giungere alla formulazione di una norma inespressa.

Analizzando nel dettaglio i vari risvolti che può assumere il discorso giuridico, il primo caso che si è immaginato è quello in cui la comprensione delle disposizioni sia "immediata" in quanto dalla loro lettura risulta chiaramente ciò che il giudice debba fare: si ritiene, in questa ipotesi, che non sia necessaria alcuna interpretazione (in claris non fit interpretatio). Se il contenuto di una disposizione normativa è univoco (ad esempio, "è vietato fumare negli edifici della Pubblica Amministrazione"), la distinzione tra disposizione e norma rimane soltanto a livello ideale, dato che tutti i destinatari del messaggio comprendono in maniera non equivoca il significato che si intende esprimere ${ }^{105}$.

Veniamo, dunque, alle altre ipotesi, a partire da quella in cui la risposta ad una determinata domanda non sia possibile poiché il giudice non trova nell'ordinamento una norma applicabile alla fattispecie: in questo caso, come si è detto, si parla di "lacuna normativa". Il processo comunicativo si complica, la comprensione del diritto oggettivo non è più "immediata", ma richiede un ragionamento più o meno complesso ed articolato che porti all'individuazione di una regola di comportamento

possibilità che nel linguaggio normativo, così come nei discorsi concernenti altri campi scientifici, si presentino lacune ed antinomie, senza che ciò comporti il "crollo" del sistema scientifico: ivi p. 362.

103 Cfr. E. BULYGIN. Il Positivismo giuridico, cit., p. 64.

104 La risposta dovrebbe essere differenziata a seconda degli ordinamenti che si prendono in considerazione, dal momento che, in linea teorica, il giudice potrebbe "non esprimersi" ed evitare di "creare" una nuova norma. Cionondimeno, nell'ordinamento italiano (art. 12 delle "preleggi" del Codice civile) il giudice è tenuto comunque a far ricorso alle «disposizioni che regolano casi simili o materie analoghe» oppure «ai principi generali dell'ordinamento giuridico» nell'ipotesi in cui non vi sia una disposizione specifica che regoli la fattispecie concreta.

Questo tipo di risposta al problema delle "lacune normative" è condiviso da diversi ordinamenti ed è coerente con la tradizione liberale dello Stato di diritto. Il codice napoleonico, infatti, allo scopo di impedire che il giudice facesse ricorso a riferimenti normativi "esterni" (in particolare alla tradizione giuridica legata al periodo precedente la Rivoluzione ) all'art. 4 stabiliva che «il giudice che rifiuterà di giudicare, con il pretesto del silenzio, dell'oscurità, o dell'insufficienza della legge, potrà essere perseguito come colpevole di diniego di giustizia». Da queste basi, sorgerà la tradizione interpretativa del "corredato disposto" e del riferimento "alle intenzioni del legislatore", quali strumenti ulteriori finalizzati a chiarire il significato delle disposizioni.

105 Non si esclude, ovviamente, che eventuali difficoltà potranno sorgere dal punto di vista "pratico", ad esempio, rispetto alla possibilità di qualificare un determinato luogo o edificio (o determinati parti di luoghi o edifici), come "pubblici" e, quindi, protetti dal divieto di fumo. E' chiaro che però in tali casi, la questione non riguarda la regola di comportamento, bensì quella (costitutiva) che individua in astratto gli ambienti a cui è attribuibile la qualità giuridica "pubblici". 
"inespressa". A meno che il giudice non voglia creare in modo del tutto arbitrario la disciplina del caso concreto ${ }^{106}$, normalmente farà uso dell" argomento analogico". Si inizia, pertanto, con I'individuare altre preposizioni normative che, secondo l'interprete o l'opinione dei giuristi, abbiano una ratio compatibile con il caso in esame. Normalmente si farà riferimento agli aspetti principali della fattispecie astratta disciplinata da queste norme ${ }^{107}$ : qualora si risconti un profilo rilevante o essenziale, si estende la medesima disciplina, introducendo nell'ordinamento una nuova norma con un medesimo contenuto deontico ma una differente premessa "fattuale". L'accostamento ad una fattispecie "legale" o all'altra, ad ogni modo, è la risultante degli argomenti sviluppati dall'interprete nella motivazione, alcuni dei quali saranno favorevoli mentre altri contrari all'analogia. È evidente, e più avanti si ritornerà sul punto, il ruolo strumentale che in ambito interpretativo ha "I'argomentazione" rispetto al "discorso giuridico" e, quindi, alla scelta delle "premesse normative" che giustificano il provvedimento preso in concreto.

In maniera analoga, in caso di "antinomia" tra due o più norme (N1 e N2), ricavate da una medesima disposizione (D) o da due disposizioni diverse (D e D1), il ragionamento del giurista non potrà che concludersi con la "decisione", circa il risultato interpretativo (N1 ○ N2) ritenuto più coerente con il "contesto normativo che si prende a riferimento"108. Dato che in ambedue i casi la scelta del contesto proposizionale dipende dal giurista, non è escluso che egli possa prendere in considerazione "disposizioni di principio" o tesi dogmatiche sulla ratio delle disposizioni della materia oggetto della controversia, uscendo pertanto da quelle che sono le interpretazioni ricavabili dagli usi linguistici e dalle regole di utilizzo dei concetti. La proposizione di partenza, dunque, viene idealmente "riscritta" in due o più modi alternativi ${ }^{109}$ e l'operatore giuridico non potrà far altro che una ricognizione degli argomenti a favore dell'uno piuttosto che dell'altro significato.

È opportuno ricordare che non sempre tra le norme e le disposizioni vi sia un rapporto "unouno": questa è una percezione elementare del momento applicativo, ma che, tuttavia, si realizza soltanto in casi eccezionali. Come si è visto sopra, all'interno del discorso giuridico in cui si forma la regola relativa alla fattispecie concreta, intervengono una molteplicità di enunciati, cosicché, di volta in volta, la norma giuridica (generale ed astratta) può essere identificata con il significato, ossia il contenuto di senso, di un singolo enunciato normativo o di un segmento di enunciati normativi o di una combinazione di enunciati normativi. In quest'ultimo caso, il linguaggio del legislatore viene "completato" dal lavoro interpretativo del giurista che ricava dall'ordinamento una norma inespressa e colma una lacuna del linguaggio normativo ${ }^{110}$.

L'individuazione della norma inespressa, invero, può derivare da diversi tipi di ragionamento, più o meno "stringenti" a seconda del tipo di connessione logica che si instaura tra gli enunciati che formano le premesse e le conclusioni dell'argomentazione.

In alcune ipotesi il ragionamento dal quale deriva la norma inespressa ha "struttura deduttiva": la conclusione è $106 \mathrm{Si}$ tenga presente quanto si è detto sopra a proposito della "formazione" e dello "sviluppo" del discorso giuridico: il primo strumento di interpretazione del linguaggio del legislatore sono gli usi linguistici e le regole semantico-lessicali comuni, nonché quelle indicate dall'autorità normativa stessa o ricavate dalla scienza giuridica.

107 Cfr. R. GUASTINI, Le fonti del diritto, cit., p. 351.

108 « [...] di fatto, rispetto alle questioni antinomiche, il principio della certezza del diritto è continuamente violato, perché in pratica il giudice accetta, di fronte ad una preposizione indecisa, ora la risposta negativa, ora quella positiva», vedi N. BOBBIO. Scienza giuridica e analisi del linguaggio, cit., p. 364.

109 Cfr. G.U. RESCIGNO. Comunicare, comprendere, interpretare nel diritto, cit., p. 706, il quale offre come esempio di contraddizione normativa, le divergenze che storicamente si sono registrare rispetto al significato dell'art. 59 Cost.: «affinché emergano le due interpretazioni alternative è necessario riscrivere idealmente le preposizioni di partenza».

$110 \mathrm{Cfr}$. N. BOBBIO. Scienza giuridica e analisi del linguaggio, cit., p. 357-8. Qui si adotta un'accezione "ampia" di lacuna normativa che viene a coincidere con ogni ipotesi in cui il linguaggio del legislatore non sia "rigoroso", ossia non elimini al massimo le probabilità di incomprensione. D'altra parte è naturale che il legislatore non tragga «dalle preposizioni normative espresse tutte le conseguenze normative che sono da esse ricavabili mediante la pura e semplice combinazione delle preposizioni in base alle regole di trasformazione ammesse come lecite», ivi p. 356-357.

111 Cfr. R. GUASTINI, Il diritto come linguaggio, cit., p. 190. Secondo I'A. «i ragionamenti deduttivi sono altresì "cogenti" o "stringenti", nel senso che, se si accettano le premesse, non si può (senza 
attraverso l'argomentazione dell'interprete, assume un linguaggio più rigoroso e completo ${ }^{112}$ : siamo al di fuori, dunque, dalle tre ipotesi di incertezza applicativa.

Non sempre, tuttavia, tra gli enunciati che formano le premesse si rinvengono soltanto disposizioni del linguaggio legislativo. In diversi casi, al fine di raggiungere "risultati" ulteriori rispetto a quelli che sarebbero ottenibili secondo la logica grammaticale, il discorso giuridico si "arricchisce" di tesi dottrinali riguardanti il significato di una determinata disposizione o di un insieme di disposizioni. Ad esempio, I'art. 40 della Cost., secondo autorevoli esponenti della dottrina, riconosce un "diritto di libertà"113: per questo motivo si sostiene che lo Stato non può né punire né vietare l'astensione collettiva dal lavoro e tantomeno può permettere che venga licenziato il lavoratore o i lavoratori che vi abbiano partecipato. Così, ad esempio, la Corte Costituzionale, nella sent. 29/1960, a partire da una disposizione costituzionale espressa, da un lato, e dalle tesi dogmatiche prevalenti, dall'altro, ha "dedotto" I'illegittimità dell'art. 502 del codice penale che puniva lo sciopero e la serrata per fini contrattuali.

Infine, possono darsi dei casi in cui il ragionamento del giudice, pur avendo ad oggetto enunciati normativi, non assuma, tuttavia, alcuno schema logico, in modo che le conclusioni a cui giunge possono essere rifiutate (senza contraddizione) pur accettando le premesse normative ${ }^{114}$. Si tratta di tecniche argomentative accettate nell'ambito della scienza giuridica e talvolta, come nel caso dell'analogia, anche dalla legge, ma che tuttavia non garantiscono la rigorosità delle conclusioni. Ad esempio, uno dei presupposti per l'applicazione dell'analogia è la possibilità di estendere la ratio legis di una disposizione al caso che si prende in considerazione. L'argomentazione che tenderà a dimostrare la sussumibilità della fattispecie concreta nel principio generale fondante la disposizione presa in considerazione, costituisce un discorso valido in ambito giuridico, ma non altrettanto in altri tipi di linguaggio scientifico. «Che il simile sia regolato con il simile» non è un principio logico, ma «è una convenzione del linguaggio giuridico»115. Lo stesso può concludersi rispetto ai vari casi in cui i giuristi operino interpretazioni "restrittive", "adeguatrici", "evolutive", ecc... Siamo di fronte ad argomenti convenzionalmente accettati nel linguaggio normativo, attraverso i quali le preposizioni normative sono "manipolate" al fine di ricavare le premesse normative adatte al caso concreto, ma non a ragionamenti auto-evidenti di cui non si possa mettere in discussione le conclusioni.

Se la regola di comportamento puntuale e concreta, contenuta nel dispositivo della sentenza, è logicamente deducibile dalle disposizioni normative espresse indicate nella motivazione "in diritto", nonché dalla descrizione dei fatti così come risulta dagli atti processuali, allora, si potrebbe sostenere, non si è dato luogo a "creazione" o "manipolazione". Negli altri casi, invece, a seconda di ciò che si ritiene opportuno, si darà luogo alla "creazione" ex novo di proposizioni normative o ad una loro "riscrittura" che, a partire da quelle originali, escluda delle parti o ne sostituisca o aggiunga delle altre ${ }^{116}$.

Nel tentativo di offrire delle conclusioni (necessariamente) approssimative, si potrebbe sostenere che nelle operazioni di applicazione degli operatori giuridici è opportuno distinguere tra "un'attività interpretativa" ed una che andrebbe più propriamente definita "manipolativa o creativa"117.

La prima si risolve nell'identificazione dei significati che esprimono le disposizioni secondo le regole grammaticali comuni. Essa, pertanto, ha uno scopo "cognitivo" in quanto informa l'interprete che una determinata disposizione (D), sul piano linguistico, può essere "compresa" in modi differenti e, dunque, esprimere differenti contenuti prescrittivi (N1, N2, N3, ecc..) $)^{118}$.

contraddirsi) rifiutare la conclusione: le premesse "garantiscono" la conclusione»

112 Ad esempio, si prendano in considerazione due regole di comportamento: una che afferma " $i$ cittadini devono pagare le tasse" (obbligo), un'altra che afferma "i disoccupati sono esenti dal pagamento delle tasse" (permesso). Dalle due preposizioni si può dedurre una regola di comportamento più "rigorosa" che prescrive: "I cittadini lavoratori devono pagare le tasse". In questo modo, come già si è spiegato, si dà luogo ad un'opera di completamento del linguaggio normativo allo scopo di prevenire eventuali incoerenze nel momento dell'applicazione.

$113 \mathrm{Si}$ veda, in tal senso, G. GIUGNI, Diritto sindacale (2001), Bari, 2004, p. 216-7, il quale sostiene che l'art. 40 «sancisce, con immediatezza, un diritto pubblico soggettivo di libertà»

114 Ossia, «le premesse non "garantiscono" la conclusione»: vedi R. GUASTINI, Il diritto come linguaggio, cit., p. 190.

115 Vedi N. BOBBIO. Scienza giuridica e analisi del linguaggio, cit., p. 358.

116 Cfr. E. BULYGIN. II positivismo giuridico, cit., p. 14-15.

117 Cfr. G.U. RESCIGNO. Comunicare, comprendere, interpretare nel diritto, cit., p. 714.

118 Cfr. R. GUASTINI. Le fonti del diritto, cit., p. 403. 
Tuttavia, quando interpreta il diritto, il giurista o l'organo dell'applicazione compie un attività "creativa" e non semplicemente "cognitiva": raramente il giurista, e tanto meno l'organo dell'applicazione, procederà all'illustrazione dei risultati interpretativi possibili senza sceglierne uno che, secondo la sua argomentazione, risulti coerente con il contesto normativo e, dunque, con l'insieme degli enunciati dell'autorità normativa e delle tesi dogmatiche della scienza giuridica che ha preso in riferimento. All'interno della "cornice" di significati (N1, N2, N3) ricavati dall'interpretazione cognitiva, l'operatore giuridico deciderà la norma (ad esempio, N2) che utilizzerà come giustificazione del provvedimento concreto. Tra l'asserto che "prescrive" (N2) e quello che "afferma ciò che il diritto dispone" (D), la differenza, prima ancora che sul piano sintattico e semantico, sta nel semplice fatto che si tratta di due enunciati diversi, anche qualora si sostenga che siano connessi da un'implicazione logica ${ }^{119}$. In questo senso, si può affermare che vi sia una "creazione in senso debole", di diritto oggettivo ${ }^{120}$.

Infine, può darsi anche la scelta dell'operatore giuridico ricada al di fuori della "cornice" di significati ricavabile secondo le tecniche interpretative in uso. In questo caso sono due gli elementi che vengono in rilievo: in primo luogo, il fatto che in tal modo si procede alla creazione di una norma "inespressa", in quanto non ricavabile dalla disposizione (D) di cui si procede all'applicazione; in secondo luogo, la norma risultante (poniamo, N4), costituisce una "creazione in senso forte" del tutto analoga ad un qualsiasi atto di produzione normativa ${ }^{121}$. Da parte sua l'interprete potrà argomentare che in realtà la manipolazione venga compiuta allo scopo di applicare una certa diposizione: egli nega di voler prescrivere un qualcosa, ma afferma di voler semplicemente spiegare il significato di una disposizione. In altro caso, al contrario, si dichiara l'intento di aggiungere, sostituire, rimuovere dei frammenti linguistici, allo scopo di adeguare l'applicazione della disposizione a determinati principi di giustizia, facendo così entrare nel ragionamento le teorie dei giuristi. Pertanto, in quest'ultima ipotesi, non si argomenta al fine di raggiungere un significato, bensì al fine di creare una diversa preposizione dalla quale si possa estrarre una norma che prescriva qualcosa di diverso e che non era ricavabile dalla preposizione originaria ${ }^{122}$.

\section{L'INTERPRETAZIONE COME "SCOPERTA" E COME “CREAZIONE".}

Nel nostro ordinamento soltanto la Corte Costituzionale potrebbe legittimamente praticare il tipo di operazioni di cui si è appena parlato. Pertanto, di fronte ad un caso concreto, a meno che non sussistano "lacune" o "contraddizioni" tra le disposizioni, I'ordinamento non permetterebbe ai giudici comuni di "manipolare" le premesse normative.

I giudici sarebbero quindi "obbligati" ad applicare quanto il diritto dispone nel caso in cui l'ordinamento detti in maniera univoca disposizioni relative al caso concreto.

Cionondimeno, occorre puntualizzare alcuni aspetti della questione al fine di evitare fraintendimenti. Anzitutto, I'obbligatorietà, come si è detto finora, è un attributo che attiene al linguaggio prescrittivo, ossia alla norma giuridica, e non alle preposizioni normative che sono invece enunciati del linguaggio descrittivo. Pertanto, un problema è quello dell'identificazione delle disposizioni applicabili al caso concreto (ricerca), un altro è quello dell'applicazione di tali disposizioni da parte del giudice o di altre autorità (che richiede un'analisi del linguaggio) ${ }^{123}$.Come è stato detto in apertura, per l'identificazione del diritto oggettivo non è sufficiente un'indagine semantica: affinché si possa dire di una disposizione che essa "appartiene" all'ordinamento giuridico e sia, perciò, "esistente", è necessario che sia effettivamente usata per prescrivere (indagine pragmatica).

Il diritto "vivente" non può essere logicamente dedotto dal testo delle preposizioni, nemmeno nel caso in cui esse presentino un testo privo di ambiguità, dal momento che l'univocità semantica non coincide e non garantisce l'effettività della norma giuridica. È pacifico che da tale problema possano derivarne altri di carattere dogmatico, quali l'invalidità (materiale) delle norme stesse in quanto ricavate in contraddizione con le premesse testuali della disposizione o con le norme sovraordinate ${ }^{124}$.

119 Cfr. E. BULYGIN. Il positivismo giuridico, cit., p. 41.

120 Cfr. R. GUASTINI. Le fonti del diritto, cit., p. 403.

121 Ibidem.

122 Cfr. G.U. RESCIGNO. Comunicare, comprendere, interpretare nel diritto, cit., p. 724-725.

123 Cfr. E. BULYGIN, Il positivismo giuridico, cit., p. 63.

124 Si ribadisce, quindi, che l'effettività della norma non dipende dalla sua validità: nell'ordinamento giuri- 
La possibilità di un simile sviamento rispetto a ciò che dal linguaggio del legislatore appare logico ricavare è data dal fatto che l'attività interpretativa è concepita in astratto come operazione finalizzata all'identificazione delle norme (astratte) che costituiscono, da un lato, «le premesse normative di una decisione giudiziale (interpretazione giudiziale)», dall'altro, «la base assiomatica di un qualche [...] sistema normativo (interpretazione in astratto dottrinale) ${ }^{125}$. L'attività giurisdizionale, in senso stretto, può essere individuata nel semplice "provvedere", ossia nello stabilire una conseguenza giuridica, ex post facto, riguardo ad eventi e circostanze già verificatesi. Tuttavia, in senso più ampio, I'operato del giudice si estende alla ricerca di una coerenza tra ciò che viene disposto e l'ordinamento nel suo complesso: in questo senso, si presuppone che il giudice debba dare giustificazione razionale del perché di fronte a determinate circostanze ha scelto quella determinata disposizione ${ }^{126}$. Và da sé che le esigenze di coerenza dell'ordinamento mettono in campo sia i criteri di organizzazione delle fonti di cui si è già visto (validità materiale, formale e criterio temporale ${ }^{127}$ ), sia le regole relative all'utilizzo dei concetti e alle derivazione di nuove "preposizioni normative" a partire da quelle originarie. In altre parole, I'analisi del linguaggio non potrà non tener conto, oltre alla logica di "formazione" del discorso giuridico, anche della gerarchia formale e materiale che i giuristi costruiscono a partire dal linguaggio giuridico stesso.

Riprendendo quanto detto sopra, se consideriamo la norma giuridica (astratta) come un atto linguistico e, pertanto, come un enunciato inseribile in un sistema di comunicazione dotato di regole convenzionali che presiedono la "formazione" e la "riproduzione" della comunicazione stessa, allora non sembra potersi dubitare che la coerenza dell'ordinamento dipende dalla "chiarezza" delle espressioni del legislatore, ossia dalla sua capacità di eseguire o meno le regole del discorso.

Il significato delle preposizioni normative sarà ricavabile, da un lato, mediante gli usi linguistici comuni e le regole semantiche poste dall'autorità normativa ${ }^{128}$ nonché quelle sviluppate dalla scienza giuridica, dall'altro, dalla volontà e dalle finalità perseguite dall'autorità normativa nel produrre la disposizione ${ }^{129}$. L'interpretazione, dunque, circoscritta a questi rilievi, si risolverebbe in un'attività conoscitiva, dal momento che l'univocità o l'equivocità delle espressioni del legislatore emergerebbe come un dato oggettivo, mediante l'applicazione dei criteri di cui sopra. Da ciò conseguirebbe, tra I'altro, che solo nelle ipotesi di lacune o antinomie nell'ordinamento si darebbe luogo a "manipolazione" e, quindi, alla creazione di una norma a partire da una o più preposizioni linguistiche. Negli altri casi, invece, l'interpretazione porterebbe alla scoperta del significato (I'unico) della disposizione .

Tuttavia, è empiricamente riscontrabile che l'univocità semantica di una disposizione non chiude la questione dell'interpretazione, dal momento che la presenza di "lacune" nell'ordinamento non dipende soltanto dal suo contenuto normativo. L'ambiguità del diritto, dunque, non emerge unicamente sul piano della formulazione linguistica delle disposizioni, ma può dipendere anche dalla costruzione del discorso giuridico compiuta dall'interprete di volta in volta. Nel processo di attribuzione del significato potremo così distinguere un "piano semantico" ed uno "pragmatico".

Il primo, come si è visto, opera mediante le regole linguistiche e, pertanto, permette di scoprire "oggettivamente"130 I'univocità o meno di una diposizione: sotto questo punto di vista, solo alcune

dico possono "esistere" ed operare più o meno a lungo norme invalide, (ad esempio perché l'invalidità non sia stata ancora rilevata).

125 Vedi P. CHIASSONI, Presentazione, cit., p. LIV.

126 Come osserva R. GUASTINI, Il diritto come linguaggio, cit., p. 197, nella motivazione delle decisioni interpretative si distinguono una "giustificazione interna", ossia «l'insieme delle premesse per sé necessarie e sufficienti a fondare logicamente la decisione (il dispositivo della sentenza)»; e una "giustificazione esterna", cioè «l'insieme delle ulteriori premesse che sono necessarie a fondare la scelta delle premesse, che a loro volta costituiscono la giustificazione interna». Rispetto alla prima, sopra si è fatto riferimento alle argomentazioni che possono essere utilizzate nel motivare in che modo viene applicato il diritto teorico nel caso individuale (dispositivo). Nella seconda, le argomentazioni saranno utilizzate a sostegno della scelta di una determinata premessa normativa (motivazione "in diritto") e conoscitiva (motivazione "in fatto").

127 Cfr. V. CRISAFULLI, Lezioni di diritto costituzionale, II, cit., p. 207.

128 Ad esempio, I'art. 12 delle "preleggi" del codice civile.

129 Cfr. P. CHIASSONI, Presentazione, cit., p. LX.

130 Si ricordi che, secondo la concezione moderna della scienza, i concetti e le regole di trasformazione del linguaggio normativo hanno origine convenzionale e, pertanto, possono assumere un valore comunicativo più o meno rigoroso, ma non si può affermare che corrispondano a verità. Quindi si intende 
disposizioni sarebbero ambigue ${ }^{131}$.

Dal punto di vista pragmatico, invece, vengono in rilievo le considerazioni dell'interprete sulla base delle quali egli fonderà la propria decisione circa il significato da attribuire ad una determinata disposizione. Il risultato dell'interpretazione, dunque, dipende anche dalle argomentazioni messe in campo dall'operatore giuridico in merito alla coerenza e alla completezza dell'ordinamento, a prescindere che esse trovino fondamento su considerazioni razionali o morali di giustizia. Sul piano pragmatico, pertanto, tutte le disposizioni sono potenzialmente ambigue, anche se semanticamente univoche ${ }^{132}$.

Le considerazioni appena svolte, dunque, ci permettono di sostenere che le lacune dell'ordinamento giuridico non siano tanto un dato oggettivo e conoscibile, quanto piuttosto una variabile dell'interpretazione giuridica, o meglio, delle tecniche argomentative che vengono di volta in volta portate a giustificazione (esterna) della decisione circa il significato che si vuole attribuire ad una disposizione ${ }^{133}$.

Le tecniche argomentative sono gli strumenti attraverso i quali l'interprete, a seconda dei casi, "crea" o "colma" le lacune giuridiche qualora non ritenga di aver riscontrato sufficiente univocità nel linguaggio normativo. Così, ad esempio, viene "creata" una lacuna quando si interpreta un termine in senso "restrittivo" o mediante l'argomento "a contrario", escludendo così che la disposizione possa estendere la sua operatività oltre un certo numero di fattispecie (che pertanto potrebbero rimanere senza disciplina giuridica). Analogamente, con l'argomento della "dissociazione", viene introdotta all'interno della classe di fattispecie disciplinata da una disposizione, una distinzione non prevista dalla sua formulazione: la norma (astratta) finisce col regolare solo una sottoclasse di comportamenti lasciando scoperti gli altri ai quali, il senso della disposizione, sembrava pur riferirsi ${ }^{134}$.

Non è vero, dunque, che tutto ciò che appare avere una forma non equivoca sia insuscettibile di interpretazione, o meglio, di "manipolazione" da parte dell'operatore giuridico. Anche al di fuori dei casi in cui il giudice è "costretto" a creare nuovo diritto a causa delle ambiguità presenti nelle formulazioni rispetto ad un caso determinato, possono darsi delle ragioni per la sostituzione delle preposizioni giuridiche con altre di diverso significato. Il giudice "comune" può avere delle ragioni morali o prudenziali per non assolvere al suo dovere giuridico e, quindi, dare la prevalenza agli "obblighi morali" della propria coscienza, piuttosto che a quelli "giuridici" enunciati dall'ordinamento ${ }^{135}$. La decisione relativa al se e come utilizzare tali argomenti, come già visto, rappresenta il frutto di valutazioni che l'interprete compie nella propria mente in base a fattori (di ordine razionale o etico) di volta in volta differenti. In tal modo, l'interpretazione risulterebbe essere sempre un atto di "creazione", in quanto, sebbene sul piano semantico vi possa essere un'immediata comprensione del linguaggio del legislatore, è l'elemento pragmatico che definisce le premesse decisionali.

Ricapitoliamo quanto si è voluto fin qui esprimere. In primo luogo, abbiamo parlato dell'ordinamento giuridico come di un vastissimo insieme di atti linguistici, il cui "contenuto" esprime ciò che noi definiamo "diritto oggettivo". Si è aggiunto che, tuttavia, quest'ultimo non coincide a sua volta con il "diritto vivente", dal momento che la forma semantica delle disposizioni non ci dà sufficienti spiegazioni rispetto a se ed in che modo esse verranno applicate. Il profilo semantico e quello pragmatico, cioè "I'essere" dalla formulazione e il "dover essere" della decisione applicativa, convivono come "opposti necessari" in contraddizione dialettica: entrambi formano parte costitutiva del processo interpretativo, benché uno tra i due (quello pragmatico) prevalga in via di fatto e retroagisca sui risultati ottenuti in ambito semantico, confermandoli o modificandoli secondo ciò che è ritenuto opportuno dall'interprete ${ }^{136}$.

Tutto ciò, nonostante le apparenze, non è in contraddizione rispetto al dato empirico per cui si riscontra una convergenza nel tradurre le disposizioni secondo il senso che esse semanticamente esprimono: una cosa è il dato pratico, un'altra quello razionale. Mentre il secondo è il frutto

"oggettivo" (in senso lato), in quanto ricavato secondo le regole interpretative in uso.

131 Ivi, p. LXIV.

132 Ibidem.

133 Cfr. R. GUASTINI, Il diritto come linguaggio, cit., p. 183.

134 Ivi, p. 185-6.

$135 \mathrm{Cfr}$. M. ATIENZA, Los limites de la interpretacion constitucional. De nuevo sobre casos tragicos, in

Isonomia, 6, 1997, pp. 7-30.

136 Cfr. P. CHIASSONI, Presentazione, cit., p. LXV. 
dell'analisi logica del linguaggio normativo, il primo è invece la conseguenza di elementi contingenti che determinano le scelte dell'interprete ${ }^{137}$. Su questa base, dunque, si forma ciò che viene definito "diritto vivente": la ratio decidendi diviene in tal senso "fatto normativo", esattamente come l'atto che produce la norma generale ed astratta.

L'estensione del consenso raggiunto in giurisprudenza o in dottrina rispetto ad una determinata soluzione interpretativa, poi, contribuisce a dare maggiore o minore stabilità al "diritto vivente", ma non può mutare in verità le norme ricavate da una certa disposizione. A tal ragione, costituiscono un esempio tangibile i diversi orientamenti che nel corso degli anni sono stati dati a medesime disposizioni anche non equivoche dal punto di vista semantico ${ }^{138}$, a dimostrazione che il linguaggio del diritto, a differenza di quello della logica analitica, non si ponga al di fuori del tempo e delle contingenze materiali che lo caratterizzano.

La "necessità", come si è detto sopra parlando delle fonti originarie, «opera come presupposto del funzionamento o anche della creazione di una o più norme giuridiche» ${ }^{139}$. Più precisamente, essa, da un lato, sta alla base del principio di effettività e dunque della creazione del diritto attraverso le fonti originarie; dall'altro, costituisce la ragione delle convergenze riscontrabili sul piano delle prassi interpretative e dunque delle possibili "manipolazioni" a cui può andare incontro I'atto linguistico normativo.

Come è intuitivo, non si può dar conto in poche pagine del concetto di "necessità" e di come esso opera nel processo di produzione e di interpretazione del diritto (ammesso che sia possibile farlo in maniera esaustiva).

Sembra tuttavia proficuo, su tale questione, richiamare quanto detto in apertura a proposito della derivazione delle regole di comportamento da "fonti sociali". Il diritto, dunque, come le altre scienze umane (I'economia, la politica, ecc...) è parte integrante della "produzione della vita sociale", in quanto "progetta", o meglio intende progettare, la generalità dei comportamenti futuri. A meno che non si voglia affermare che una società scelga a caso il regime politico, il sistema economico, I'ordinamento giuridico, ecc... di cui intende dotarsi, si dovrà ritenere che tale scelta sia piuttosto una conseguenza dell'insieme delle domande (istanze) presenti all'interno del corpo sociale. Si è detto "insieme delle domande" non a caso: sarebbe infatti erroneo ritenere che ogni "produzione sociale" derivi in autonomia dalle istanze particolari di quel determinato settore della vita sociale a essa cui si riferisce.

Così, le disposizioni formulate dal "legislatore" possono subire delle trasformazioni, più o meno "lecite" dal punto di vista degli usi interpretativi, in sede di applicazione: nel caso in cui le parole non vengano più ritenute sufficienti per rispondere alle domande concrete l'operatore giuridico può sentire la necessità di spingersi ad indagare sulla prassi sociale al fine di dare una risposta opportuna. Per i motivi di cui si è appena detto, non sempre tale attività è agevole, dal momento che non è agevole ragionare sopra una "produzione sociale", qual è il diritto, dividendo i campi del sapere e costruendo per ciascuno di essi degli specifici rapporti di causalità attraverso i quali dare spiegazione della complessa fenomenologia che li caratterizza. «La società intesa come modo di produzione, unità complessa di istanze autonome, non può essere compresa nel suo funzionamento e nella sua evoluzione se non in riferimento alle determinazioni che emanano da tutti i livelli, da tutte le istanze. Quest'insieme di determinazioni costituisce una struttura che può essere considerata esplicativa del rapporto causale sociale» ${ }^{140}$.

137 L'interprete, pertanto, sceglierà se adottare un atteggiamento più o meno "conformista" rispetto all'orientamento interpretativo prevalente.

$138 \mathrm{~A}$ tal proposito si è prodotto l'esempio dell'art. 59 c.2 (Cfr. supra Cap I) il quale, nonostante la sua univocità semantica, sotto la presidenza Pertini è stato interpretato nel senso che ciascun Presidente della Repubblica fosse autorizzato a nominare cinque senatori a vita e non nel senso che i senatori a vita dovessero essere in tutto cinque.

Ancor più emblematica, sul medesimo punto, è la posizione della Corte Costituzionale (sent. 16/1978) rispetto all'art. 75 c.2: nonostante il testo della disposizione riporti un elenco, per cui potrebbe essere dedotta I'intenzione del Costituente di individuare in maniera tassativa le materie sulle quali non può essere proposto referendum, i giudici di legittimità ritengono che in realtà si tratti di una prescrizione a "fattispecie aperta" e, pertanto, l'elenco sarebbe suscettibile di continuo aggiornamento (come effettivamente lo è stato).

139 Vedi A. PIZZORUSSO, Delle fonti del diritto, cit., p. 11.

140 Vedi M. MIAILLE, Introduzione allo studio critico del diritto, cit., p. 72. Secondo l'A., dunque, «non è il livello economico, politico o ideologico che spiega tale o tal'altro gesto che io compio, è la struttura complessa delle causalità che appartengono a questi diversi livelli a essere la "causa" di questo gesto». 
ALCHOURRÒn C.E. Logic of Norms and Logic of Normative Propositions, in Logique et analyse, 12 , 1969.

ATIENZA M., Los limites de la interpretacion constitucional. De nuevo sobre casos tragicos, in Isonomia, 6, 1997.

BOBBIO, N. Scienza del diritto e analisi del linguaggio (1950), oggi in Contributo ad un dizionario giuridico, Torino, 1994.

BULYGIN E.; ALCHOURRON C.E. La norma giuridica, in E. BULYGIN, Il positivismo giuridico, Milano, 2007.

COLEMAN J. L. On the relationship between laws and morals, in Ratio juris, 2, 1989.

CRISAFULLI V. Lezioni di diritto costituzionale, II, Padova, 1993.

ESPOSITO C., Validità delle leggi. Studi sui limiti della potestà legislativa, i vizi degli atti legislativi e il controllo giurisdizionale, Padova, 1934.

G. GIUGNI, Diritto sindacale (2001), Bari, 2004.

GUASTINI R. Il diritto come linguaggio. Lezioni, Torino, 2006.

GUASTINI R. Le fonti del diritto. Fondamenti teorici, Milano, 2010.

MIAILLE M. Introduzione allo studio critico del diritto, Roma, 1979.

MODUGNO F. Appunti dalle lezioni sulle fonti del diritto, Torino, 2005.

PERASSI T. Necessità e stato di necessità nella teoria dommatica della produzione giuridica (1917), in Scritti giuridici, I, Milano, 1958.

PIZZORUSSO A. Corso di diritto comparato, Milano, 1983.

RAZ J. Legal reasons, sources and gaps, in The authority of law, Oxford, 1979.

RESCIGNO G.U. Comunicare, comprendere interpretare nel diritto, in Diritto pubblico, n.3/2009.

RESCIGNO G.U. L'atto normativo, Bologna, 1998.

VON WRIGHT G.H. Norma e azione. Un'analisi logica, Bologna, 1989. 